\title{
Dynamic pricing and reservation for intelligent urban parking management
}

\author{
Chao Lei ${ }^{\mathrm{a}}$, Yanfeng Ouyang ${ }^{\mathrm{a}, *}$ \\ ${ }^{a}$ Department of Civil and Environmental Engineering, University of Illinois at Urbana-Champaign, Urbana, IL 61801, \\ United States
}

\begin{abstract}
Despite rapid advances of information technologies for intelligent parking systems, it remains a challenge to optimally manage limited parking resources in busy urban neighborhoods. In this paper, we use dynamic location-dependent parking pricing and reservation to improve systemwide performance of an intelligent parking system. With this system, the parking agency is able to decide the spatial and temporal distribution of parking prices to achieve a variety of objectives, while drivers with different origins and destinations compete for limited parking spaces via online reservation. We develop a multi-period non-cooperative bi-level model to capture the complex interactions among the parking agency and multiple drivers, as well as a non-myopic approximate dynamic programming (ADP) approach to solve the model. It is shown with numerical examples that the ADP-based pricing policy consistently outperforms alternative policies in achieving greater performance of the parking system, and shows reliability in handling the spatial and temporal variations in parking demand.
\end{abstract}

Keywords: Parking management, Dynamic pricing, Approximate dynamic programming, Equilibrium, MPEC

\section{Introduction}

In recent years, rapid advances of information technologies bring in fast development of intelligent parking systems (IPS) all over the world. Inside IPS, dynamic parking pricing is an important component that makes the system "intelligent" in terms of utilizing limited parking resources. For example, in 2008, San Francisco Municipal Transportation Agency (SFMTA) launched an SFpark project, through which it successfully implemented a demand-responsive parking pricing scheme for on-street metered spaces and SFMTA managed garages in several selected areas in San Francisco (San Francisco Municipal Transportation Agency, 2014). The fundamental idea of the program is, by properly manipulating parking rates, to maintain an ideal level of occupancy in every city block, such that any newly arriving driver can always find a suitable open space without circling around for parking. Similar practices of smart parking include the so-called LA ExpressPark ${ }^{\mathrm{TM}}$ program in Downtown Los Angeles, and SeaPark in

*Corresponding author. Tel: +1 217333 9858; fax: +1 2173331924 .

Email: yfouyang@illinois.edu

Preprint submitted to Transportation Research Part C: Emerging Technologies

January 20, 2017 
Seattle. These existing programs nevertheless share a common feature: the parking prices are updated once per several weeks or even several months, which is not sensitive enough to deal with the highly dynamic parking demand in realistic daily operations. The lack of quick response would inevitably limit the effectiveness of dynamic pricing strategies.

The rapid advances of information and communication technologies have presented tremendous opportunities for smart parking; e.g., on-site detectors can track occupancy status at a parking space (Panja et al., 2011; Park et al., 2008) and instantaneously communicate that information to drivers via smartphone. The drivers can also make parking searches and reservations online in real time. The emergence of numerous parking applications on smartphone, e.g., SpotHero, ParkWhiz, ParkMe, Parking Panda, has made it possible for drivers to check the availability of parking spaces and make reservations in advance of their trips (Chen et al., 2015). Besides the fact that drivers' experiences can be greatly improved by introducing reservation service, the size of the parking market would also be potentially expanded. For instance, some first-time travelers who are unfamiliar with parking neighborhoods can now conveniently find suitable parking space through the reservation system. In addition, parking reservation service may as well have a positive effect in reducing urban congestion. Yang et al. (2013) pointed out that allowing reservation service for a portion of parking spaces is helpful to reduce congestion because it helps spread the departures of those morning commuters.

As such, the prospect of implementing dynamic parking pricing and reservation strategies has become technically feasible and socioeconomically appealing. Our study, therefore, is devoted to developing sophisticated mathematical models to maximize the performance of dynamic parking pricing strategies so as to make the best use of limited parking resources in urban areas. We attempt to implement the system through an online reservation system which allows drivers to make reservations to secure a parking spot for a certain period of time. One of the challenges associated with the parking system is that stakeholders, usually including the agency and individual drivers, exhibit certain gaming behaviors in their decision-making processes. For example, the agency uses differential pricing strategy to influence drivers parking location choice, increase parking revenue, or balance spatial distribution of parking reservations. Meanwhile, individual drivers compete for limited parking spaces at popular locations based on the parking price and their travel convenience. Hence, it is essential to come up with an overarching modeling framework to jointly consider the objectives of different stakeholders in conducting parking system management.

This paper develops a mathematical programming model for the demand-driven dynamic parking pricing and reservation problem, where drivers are allowed to make parking reservations prior to the trips and secure parking spaces for a length of future time. The problem is formulated in the form of a multi-period mathematical program with equilibrium constraints (MPEC), which is extremely difficult to solve. We further develop an effective non-myopic algorithm based on approximate dynamic programming (ADP) to solve the model, such that future parking supply and demand information is incorporated into a dynamic pricing decision-making process. An embedded subroutine is also proposed to transform the bi-level MPEC model (for each period) into a solvable single-level mixed-integer quadratic program (MIQP). The performance of the proposed model and algorithm is tested through a series of numerical experiments, and managerial insights are drawn. It is shown that, through ADP-based pricing policy, the parking system can achieve much more superior performance as compared with those under alternative policies.

The remainder of the paper is organized as follows. Section 2 first presents a literature review over parking pricing, parking management, as well as a few state-of-the-art modeling and solution methodologies. Section 3 explains the notation and formulation of the proposed parking 
pricing problem. Section 4 describes the solution algorithm based on ADP and MIQP transformation. Section 5 illustrates the numerical experiments. Finally, Section 6 concludes the paper and discusses possible directions of future research.

\section{Literature Review}

The parking management problem has attracted considerable attention in the past decade. Shoup (2005) illustrated the significant role of parking pricing in changing the landscape of modern traffic system. Some of the ideas in this monograph have inspired the SFpark project mentioned above.

Most of the existing research on parking pricing is devoted to investigating ways to use parking pricing strategies to influence drivers' competitions for parking resources. The hope is to eventually enhance the performance of the transportation system, especially for urban morning commute (Arnott et al., 1991; Lam et al., 1999; Zhang et al., 2008; Qian et al., 2011, 2012). Qian and Rajagopal (2014a) proposed a dynamic parking pricing model to achieve optimal flow pattern in a network. The day-to-day recurrent behavior of morning commuters is formulated under driver equilibrium conditions, while the drivers are assumed to always know parking price and occupancy information. The authors also studied a similar parking pricing problem under stochastic demand (Qian and Rajagopal, 2014b), and further extended the scope to investigate both departure time choices and parking location choices Qian and Rajagopal (2015). Some reservation strategies have also been proposed to mitigate parking-related delay and traffic congestion. For example, Zhang et al. (2011) and later Liu et al. (2014b) introduced a tradable parking permit scheme for the morning commuters parking management problem. Yang et al. (2013) studied a morning commute problem under the circumstance that some commuters have reserved parking spots. Later, Liu et al. (2014a) considered a parking reservation scheme with expiration, where a commuter is required to arrive at its parking space before a predetermined expiration time. Chen et al. (2015) proposed a parking reservation system to manage the allocation of downtown curbside parking spaces; a simple reservation scheme was proposed to minimize the total social cost associated with parking. Chen et al. (2016) later showed that reservation service can improve the performance of a stylized parking system where a destination of parking demand is located on a long one-way street and on-street parking spaces are scattered around the destination. However, in these studies, parking pricing as a strategy to balance spatial demand is not integrated into the reservation mechanism.

Only a small amount of literature is devoted to considering pricing as a management strategy to improve the performance of the parking system. For example, Guo et al. (2012) proposed a game theoretic based parking choice model and pointed out that a Nash equilibrium can be derived by assuming drivers make parking decisions simultaneously and have perfect knowledge about other fellow drivers' strategies. Ayala et al. $(2011,2012)$ presented a parking pricing model for the parking competition problem and examined the influence of pricing strategies on drivers' parking competition. He et al. (2015) extended Ayala et al.'s work by formulating the equilibrium state of the parking competition as a system of nonlinear equations and characterizing all the pricing schemes to reach system optimum assignment of parking spaces. These studies acknowledged the critical role of pricing in parking management. Unfortunately, the pricing strategies discussed in these studies were static in nature; e.g., parking demand was assumed to remain constant over time. In contrast, Geng and Cassandras (2011) proposed a dynamic model for optimal allocation of parking spaces, while the parking demand was assumed to be given and 
the system manager had full control over all drivers. Such a model does not capture the characteristics of drivers' competition for limited parking spaces, nor does it consider the impact of agency's pricing strategy on parking demand.

Only recently have dynamic pricing and drivers' parking behavior been incorporated into an optimization framework. Mackowski et al. (2015) developed a dynamic and demand-based pricing model to optimally manage parking resources in a busy urban neighborhood. They explicitly addressed the interactive relationship between pricing and parking demand via a dynamic MPEC model. Yet, a driver is not allowed to reserve a parking space for a length of future time prior to the trip. Also, due to intensive computational burden associated with the dynamic MPEC problem, a myopic sequential solution approach was proposed to solve the model. Such type of models, albeit useful in terms of addressing spatial allocation of parking resources, will not be able to fully utilize valuable information on future parking demand and supply (which may be crucial for temporal allocation of resources).

Over the past few decades, a growing body of studies has demonstrated that the APD is a powerful tool in handling enormous types of dynamic optimization problems with complex operational details. The ADP algorithms rely on the use of simulation techniques and parametric approximations to obtain a suitable estimate of the value function in the high-dimensional state space. With ADP, valuable information throughout the entire planning horizon can be effectively incorporated into dynamic decision-making. Interested readers are referred to the monographs by Bertsekas and Tsitsiklis (1996), Sutton and Barto (1998) and Powell (2011) for comprehensive reviews of this research field.

Meanwhile, the Stackelberg leader-follower game, often formulated in the form of MPEC models, has been widely applied to many hierarchical optimization problems, in contexts such as transportation planning (Yang and Bell, 2001; Lu, 2007) and supply chain management (Bai et al., 2012b). The MPEC formulation captures the complex interactions among multiple stakeholders' decision-making processes in an integrated way. The readers are referred to Luo et al. (1996) for a comprehensive review. Due to the non-convexity and non-smoothness nature of MPEC problems, solving them is a non-trivial task in general. The computation time grows extremely fast with the problem size, and existing solvers often have limited power to deal with relatively large instances. Nevertheless, some useful algorithms have been developed by transforming MPEC problems (with or without discrete constraints (Bai et al., 2016)) into equivalent mixed-integer linear/quadratic problems that can be better handled by solvers (Hu et al., 2008, 2011; Bai et al., 2012a).

\section{Mathematical Modeling}

In this section, we present the notation and formulation of the dynamic parking pricing and reservation problem. We consider this dynamic problem as a Stackelberg leader-follower game and formulate it as a finite-horizon multi-period bi-level MPEC model, where the agency's pricing problem is in the upper level and the drivers' collective parking choice equilibrium is in the lower level.

Consider an urban area with a set of spatially distributed parking lots $\mathcal{J}$ and each parking lot $j \in \mathcal{J}$ has a capacity $C_{j}$. The agency needs to update the parking price at discrete times in a pre-specified planning horizon. For the clarity of illustration, we call each time instant at which the agency updates the parking prices as a decision epoch, and denote all decision epochs as a set $\mathcal{T}=\{0,1,2, \ldots, T\}$ indexed by $t$. The time interval between any two consecutive decision epochs has the same length (i.e., a single time period). 
At each decision epoch, a driver is allowed to reserve an available parking space in any parking lots for a future duration within the upcoming reservation horizon. The reservation horizon starts at the current decision epoch, and its duration, measured by the number of time periods, comes from a set $\mathcal{M}=\{1,2, \ldots, M\}$. At each decision epoch $t$, the agency needs to determine the prices for each parking lot in the upcoming reservation horizon. The parking price is dependent on the decision epoch as well as the time period within a reservation horizon. As such, we let $p_{t m j}$ represent the unit price (per space) for parking in lot $j \in \mathcal{J}$ from time $t+m-1$ to time $t+m$, where $m \in \mathcal{M}$, and let $\mathbf{p}_{t}:=\left\{p_{t m j} \mid \forall m \in \mathcal{M}, \forall j \in \mathcal{J}\right\}$. A pricing decision for the entire planning horizon is then written as $\mathbf{p}=\left\{\mathbf{p}_{0}, \mathbf{p}_{1}, \ldots, \mathbf{p}_{T}\right\}$. In practice, parking prices are often subject to certain restrictions enforced by city legislations. In this work, we impose constraints (1)-(3) as the restrictions over parking price.

$$
\begin{aligned}
& P_{j}^{L} \leq p_{t m j} \leq P_{j}^{U}, \quad \forall j \in \mathcal{J}, m \in \mathcal{M}, t \in \mathcal{T}, \\
& -\epsilon_{l} \leq p_{t, m, j}-p_{t, m-1, j} \leq \epsilon_{r}, \quad \forall j \in \mathcal{J}, m \in \mathcal{M} \backslash\{0\}, t \in \mathcal{T}, \\
& -\epsilon_{l} \leq p_{t+1, m-1, j}-p_{t, m, j} \leq \epsilon_{r}, \quad \forall j \in \mathcal{J}, m \in \mathcal{M} \backslash\{0\}, t \in \mathcal{T} \backslash\{T\} .
\end{aligned}
$$

Constraints (1) enforce a lower limit $P_{j}^{L}$ and an upper limit $P_{j}^{U}$ over the price in lot $j \in \mathcal{J}$. Constraints (2) state that the price fluctuation between two consecutive time periods in a reservation horizon is restricted within the range of $\left[-\epsilon_{l}, \epsilon_{r}\right]$, and constraints (3) state that the price fluctuation between two consecutive decision epochs must be restricted within $\left[-\epsilon_{l}, \epsilon_{r}\right]$ as well.

For the simplicity of modeling, we categorize the users into different types and consider the flow of each type of users as the parking demand. The type of parking demand is specified by the origin-destination $o d \in O \mathcal{D}$, the arrival time of reservation $t \in \mathcal{T}$, the start time of parking $t+t_{s}-1$ with $t_{s} \in \mathcal{M}$, and the parking duration $\tau \in \Gamma_{t_{s}}$, where $\Gamma_{t_{s}}=\left\{1, \ldots, M+1-t_{s}\right\}$. Here we use the index combination $\left(t, o d, t_{s}, \tau\right)$ to represent a specific demand type in the paper. The amount of demand for each type follows an inverse demand function $H(\cdot)$ of the parking disutility $u_{t, o d, t_{s}, \tau}$. In this work, we assume that $H(\cdot)$ is a linear non-increasing function, ${ }^{1}$ i.e., $H\left(u_{t, o d, t_{s}, \tau}\right)=a_{t, o d, t_{s}, \tau}-b_{t, o d, t_{s}} u_{t, o d, t_{s}, \tau}$, where $a_{t, o d, t_{s}, \tau}$ denotes the intercept of the demand curve and $b_{t, o d, t_{s}}$ captures the demand elasticity. The parking disutility $u_{t, o d, t_{s}, \tau}$ is measured by the summation of parking payment, two-way travel cost between the origin and the parking lot, and two-way walking cost between the parking lot and the destination. The total parking payment for a demand of type $\left(t, o d, t_{s}, \tau\right)$ parking in lot $j$ is calculated as $\sum_{m=t_{s}}^{t_{s}+\tau-1} p_{t m j}$. For simplicity of notation, we combine the travel cost and the walking cost between OD pair $o d$ and parking lot $j$ into one term, $\varphi_{t, o d, j}$.

(Place Figure 1 about here).

We define $h_{t, o d, t_{s}, \tau, j}$ as the amount of parking spaces in lot $j$ that are reserved by the demand of type $\left(t, o d, t_{s}, \tau\right)$, as shown in Figure 1 . Due to the limited capacity of parking lots, it is possible that not all the reservations can be accommodated by the system. Hence, we introduce a dummy lot $\zeta$ to absorb all the excessive demand $h_{t, o d, t_{s}, \tau, \zeta}$. We now have the market clearing constraints

\footnotetext{
${ }^{1}$ Due to its simplicity, the linear demand function is one of the most widely used models for responsive demand (Hobbs et al., 2000; Bai et al., 2012b; Mackowski et al., 2015). It allows us to reformulate the bilinear term in the objective function into an equivalent convex quadratic and linear form, as shown in Section 4.3. Since we explicitly restrict the fluctuation of parking prices, even when real-world demand model is nonlinear, we can probably use local linearization as an approximation.
} 
as follows:

$$
H\left(u_{t, o d, t_{s}, \tau}\right)=\sum_{j \in \mathcal{T} \cup \zeta} h_{t, o d, t_{s}, \tau, j}, \quad \forall o d \in O \mathcal{D}, t_{s} \in \mathcal{M}, \tau \in \Gamma_{t_{s}}, t \in \mathcal{T} .
$$

We denote the occupancy of the parking lots at decision epoch $t$ by vector $\mathbf{f}_{t}=\left[f_{t m j}\right]_{\forall m \in \mathcal{M}, \forall j \in \mathcal{T}}$, where $f_{t m j}$ is the number of vacant spaces in lot $j$ from time $t+m-1$ to time $t+m$. As shown in Figure 1, the process of the dynamic pricing and reservation can be described as follows: at decision epoch $t \in \mathcal{T}$, given the available parking space at lot $j \in \mathcal{J}$ in the upcoming reservation horizon from time $t$ to time $t+M$, the agency determines the parking price $p_{t m j}$ from time $t+m-1$ to time $t+m$, where $m \in M$. Meanwhile, the users of different types reserve available parking spaces in parking lot $j$ for a future duration within the upcoming reservation horizon. After the reservation of decision epoch $t$, the parking spaces that are left empty are then passed to the next reservation horizon at decision epoch $t+1$. Therefore, the following conservation constraints should be satisfied:

$$
\begin{aligned}
& f_{t+1, m-1, j}=f_{t m j}-\sum_{o d} \sum_{t_{s}=1}^{m} \sum_{\tau=m+1-t_{s}}^{M+1-t_{s}} h_{t, o d, t_{s}, \tau, j}, \quad \forall j \in \mathcal{J}, m \in \mathcal{M} \backslash\{1\}, t \in \mathcal{T} \backslash\{T\}, \\
& f_{t+1, M, j}=C_{j}, \quad \forall j \in \mathcal{J}, t \in \mathcal{T} \backslash\{T\} .
\end{aligned}
$$

Constraints (5) represent that the currently available parking spaces in lot $j$ are either occupied by the newly arrival demand or passed to the next reservation horizon. Constraints (6) state that each parking lot is restored to its full capacity by the end of the last period.

In the upper level, the parking agency acts as a leader and aims at maximizing the total benefit over the entire planning horizon as follows.

$$
\max _{\mathbf{p}}\left\{\sum_{t=0}^{T} \gamma^{t} G_{t}\left(\mathbf{f}_{t}, \mathbf{p}_{t}\right) \mid \overline{\mathbf{f}}_{0}\right\},
$$

where $\overline{\mathbf{f}}_{0}$ denotes the initial occupancy level of the parking lots, $\gamma$ denotes the discounting factor, and $G_{t}\left(\mathbf{f}_{t}, \mathbf{p}_{t}\right)$ denotes the objective function of the agency at decision epoch $t$, which is defined as:

$$
\begin{aligned}
G_{t}\left(\mathbf{f}_{t}, \mathbf{p}_{t}\right)=\alpha_{1}\left\{\sum _ { o d } \sum _ { t _ { s } } \sum _ { \tau = 1 } ^ { M + 1 - t _ { s } } \sum _ { j } \left[\sum_{m=t_{s}}^{t_{s}+\tau-1} p_{t m j} h_{t, o d, t_{s}, \tau, j}\right.\right. \\
\left.\left.\quad+\frac{1}{2} h_{t, o d, t_{s}, \tau, j}\left(u_{t, o d, t_{s}, \tau}^{\max }-u_{t, o d, t_{s}, \tau}\right)\right]\right\}-\alpha_{2}\left(\sum_{j} \beta\left|\kappa_{j} C_{j}-f_{t, 1, j}\right|\right),
\end{aligned}
$$

where $u_{t, o d, t_{s}, \tau}^{\max }$ denotes the parking disutility when the parking demand for this type of users reduces to zero, i.e., $u_{t, o d, t_{s}, \tau}^{\max }=H^{-1}(0)$. The objective function (8) states that the agency aims at maximizing the total economic surplus while minimizing the occupancy imbalance penalty. $\alpha_{1}$ and $\alpha_{2}$ are the respective weights for these two terms. The total economic surplus, shown as the first two terms in (8), includes the agencys parking revenue and the consumer surplus. From Figure 2, we can see that the parking revenue is the total parking payments that the agency collects, and the consumer surplus is the difference between the total amount of money that customers are able to pay and the total amount that they actually do pay. 
(Place Figure 2 about here).

The last term in (8) presents the spatial balance objective. We assume that a penalty cost would be incurred if a parking lot fails to meet its target vacancy level. The occupancy imbalance penalty is computed as the product of the penalty cost factor $\beta$ and the absolute difference between the remaining parking spaces in each parking lot and its target vacancy level $\kappa_{j} C_{j}$, where $\kappa_{j}$ denotes the target percentage.

In the lower level, each individual parking driver acts as a follower and makes his/her own optimal parking decision while competing against others for limited parking spaces. We assume that all the parking drivers are rational users, who are in pursuit of minimizing their parking disutilities, and they make parking reservations simultaneously at each decision epoch through online reservation systems. Hence, we consider that the drivers parking behaviors satisfy a type of Nash equilibrium in transportation networks (Sheffi, 1985). The parking disutility $u_{t, o d, t_{s}, \tau}$ is then regarded as the equilibrium disutility for users of type $\left(t, o d, t_{s}, \tau\right)$. As such, the agency's pricing scheme can be considered to be effective in adjusting the demand. According to Facchinei and Pang (2004) and Pang and Fukushima (2005), the existence of equilibrium at each decision epoch can be regarded as the existence of a solution to the generalized Nash equilibrium (GNE) for each subproblem.

Let $\phi_{t, o d, t_{s}, \tau, j}=\sum_{m=t_{s}}^{t_{s}+\tau-1} p_{t m j}+\varphi_{t, o d, j}$ denote the disutility of demand of type $\left(t, o d, t_{s}, \tau\right)$ at lot $j$. Due to the assumption that the demand can be assigned to the dummy lot only if there is no available spaces in any of parking lots, the disutility for using dummy lot must be set higher than the disutilities for using the regular parking lots but no higher than the intercept of the demand curve. Thus, the disutility for type $\left(t, o d, t_{s}, \tau\right)$ demand using dummy lot is defined as:

$$
\Phi_{t, o d, t_{s}, \tau, \zeta}=\min \left\{H^{-1}(0), \max _{j \in \mathcal{J}}\left\{\sum_{m=t_{s}}^{t_{s}+\tau-1}\left(p_{t-1, m+1, j}+\epsilon_{r}\right)+\varphi_{o d, j}\right)\right\} .
$$

Let $I_{t, o d, t_{s}, \tau}$ denote the set of type $\left(t, o d, t_{s}, \tau\right)$ users. We define the parking decision of an individual user $i \in I_{t, o d, t_{s}, \tau}$ as:

$$
z^{i j}= \begin{cases}1, & \text { if user } i \text { chooses to park in lot } j \\ 0, & \text { otherwise. }\end{cases}
$$

Given the value of $\phi_{t, o d, t_{s}, \tau, j}, \forall j \in \mathcal{J} \cup \zeta$, the parking optimization problem for user $i \in I_{t, o d, t_{s}, \tau}$ can be written as follows:

$$
\begin{array}{ll}
\min & \sum_{\forall j \in \mathcal{J} \cup\{\zeta\}} \phi_{j} z^{i j} \\
\text { s.t. } & \sum_{\forall j \in \mathcal{J} \cup\{\zeta\}} z^{i j}=1, \\
& z^{i j}+\sum_{i^{\prime} \in \tilde{I}_{t}^{m}, i^{\prime} \neq i,} z^{i^{\prime} j} \leq f_{t m j}, \quad \forall j \in \mathcal{J}, m \in \mathcal{M}_{t_{s}, \tau}^{\prime}, \\
& z^{i j} \in\{0,1\}, \quad \forall j \in \mathcal{T} \cup\{\zeta\},
\end{array}
$$

where $\mathcal{M}_{t_{s}, \tau}^{\prime}=\left\{t_{s}, \ldots, t_{s}+\tau-1\right\}$, and $\tilde{I}_{t}^{m}$ denotes the set of users whose reservations arrive at $t \in \mathcal{T}$ and having overlaps with each other at period $m \in \mathcal{M}$, i.e., $\tilde{I}_{t}^{m}=\left\{i \in I_{t, o d, t_{s}, \tau} \mid \forall o d \in\right.$ $\left.O \mathcal{D}, t_{s} \in\{1, \ldots, m\}, \tau \in\left\{m+1-t_{s}, \ldots, M+1-t_{s}\right\}\right\}$. 
The objective function (9) is to minimize the user's parking disutility. Constraint (10) ensures that each user must select a space, either in one of the regular parking lot or in the dummy lot. Constraints (11) enforce the capacity limits over the parking lots. Note that all other users whose demand has spatial or temporal overlaps with that of user $i$ are coupled together in constraints (11). Constraints (12) state the binary nature of the decision variables. Nevertheless, as problem (9)-(12) fits the special structure of totally unimodular assignment problem, the binary constraints (12) can actually be relaxed into the following:

$$
0 \leq z^{i j} \leq 1, \quad \forall j \in \mathcal{J} \cup\{\zeta\}
$$

Now we attempt to derive the equilibrium constraints for the lower level problem in the form of Karush-Kuhn-Tucker (KKT) conditions (Bai et al., 2012b, 2016). Denote $\lambda_{i}, \rho_{m j}$ and $\mu_{i j}$ as dual multipliers with respect to constraints (10), (11) and (13), then the Lagrangian function for problem (9)-(12) is:

$$
\mathcal{L}(\lambda, \rho, \mu)=\sum_{j} \phi_{j} z_{i j}+\sum_{j} \mu_{i j}\left(z_{i j}-1\right)-\lambda_{i}\left(\sum_{j} z_{i j}-1\right)+\sum_{m} \sum_{j} \rho_{m j}\left(Z_{j}-f_{t m j}\right),
$$

where $Z_{j}=z^{i j}+\sum_{i^{\prime} \in \tilde{I}_{t}^{m}, i^{\prime} \neq i,} z^{i^{\prime} j}$.

We can now write down the first-order KKT conditions of the optimization problem (9)-(12) as follows:

$$
\begin{aligned}
& 0 \leq z_{i j} \perp\left(\phi_{j}+\mu_{i j}-\lambda_{i}+\sum_{m \in \mathcal{M}_{t s, \tau}^{\prime}} \rho_{m j}\right) \geq 0, \quad \forall j \in \mathcal{J}, \\
& 0 \leq z_{i j} \perp\left(\phi_{j}+\mu_{i j}-\lambda_{i}\right) \geq 0, \quad j=\zeta, \\
& 0 \leq \mu_{i j} \perp\left(z_{i j}-1\right) \geq 0, \quad \forall j \in \mathcal{J} \cup\{\zeta\}, \\
& 0 \leq \lambda_{i} \perp\left(\sum_{j} z_{i j}-1\right) \geq 0, \\
& 0 \leq \rho_{m j} \perp\left(f_{t m j}-Z_{j}\right) \geq 0, \quad \forall j \in \mathcal{J}, \forall m \in \mathcal{M}_{t_{s}, \tau}^{\prime},
\end{aligned}
$$

where operator $\perp$ indicates the complementarity condition, i.e., $0 \leq a \perp b \geq 0 \Leftrightarrow a b=0$ and $a \geq 0, b \geq 0$.

Knowing that $\mu_{i j}=0$ is always feasible to the above KKT conditions, we can simplify (14) $\sim(16)$ as:

$$
\begin{aligned}
& 0 \leq z_{i j} \perp\left(\phi_{j}-\lambda_{i}+\sum_{m \in \mathcal{M}_{t s, \tau}^{\prime}} \rho_{m j}\right) \geq 0, \quad \forall j \in \mathcal{J}, \\
& 0 \leq z_{i j} \perp\left(\phi_{j}-\lambda_{i}\right) \geq 0, \quad j=\zeta, \\
& z_{i j}-1 \leq 0, \quad \forall j \in \mathcal{J} \cup\{\zeta\} .
\end{aligned}
$$

Based on the postulation that $\lambda_{i}=\lambda_{i^{\prime}}, \forall i^{\prime} \in I_{t, o d, t_{s}, \tau}$, we can sum up (19) and (20) over $I_{t, o d, t_{s}, \tau}$ and 
obtain:

$$
\begin{aligned}
& 0 \leq h_{j} \perp\left(\phi_{j}-u+\sum_{m \in \mathcal{M}_{t_{s}, \tau}^{\prime}} \rho_{m j}\right) \geq 0, \quad \forall j \in \mathcal{J}, \\
& 0 \leq h_{j} \perp\left(\phi_{j}-u\right) \geq 0, \quad j=\zeta,
\end{aligned}
$$

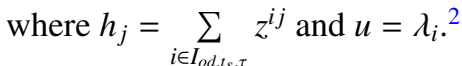

Therefore, we successfully obtain the lower level equilibrium constraints for each decision epoch $t$ :

$$
\begin{aligned}
& 0 \leq h_{t, o d, t_{s}, \tau, j} \perp\left(\phi_{t, o d, t_{s}, \tau, j}+\sum_{m \in \mathcal{M}_{t s, \tau}^{\prime}} \rho_{t m j}-u_{t, o d, t_{s}, \tau}\right) \geq 0, \\
& \forall o d \in O \mathcal{D}, t_{s} \in \mathcal{M}, \tau \in \Gamma_{t_{s}}, j \in \mathcal{J}, t \in \mathcal{T}, \\
& 0 \leq h_{t, o d, t_{s}, \tau, \zeta} \perp\left(\Phi_{t, o d, t_{s}, \tau, \zeta}-u_{t, o d, t_{s}, \tau}\right) \geq 0, \quad \forall o d \in O \mathcal{D}, t_{s} \in \mathcal{M}, \tau \in \Gamma_{t_{s}}, j=\zeta, t \in \mathcal{T}, \\
& 0 \leq \rho_{t m j} \perp\left(f_{t m j}-\sum_{o d \in O \mathcal{D}} \sum_{t_{s}=1}^{m} \sum_{\tau=m+1-t_{s}}^{M+1-t_{s}} h_{t, o d, t_{s}, \tau, j}\right) \geq 0, \quad \forall j \in \mathcal{J}, \forall m \in \mathcal{M}, t \in \mathcal{T}, \\
& 0 \leq u_{t, o d, t_{s}, \tau} \perp\left(\sum_{j \in \mathcal{J} \cup \zeta} h_{t, o d, t_{s}, \tau, j}-H\left(u_{t, o d, t_{s}, \tau}\right)\right) \geq 0, \quad \forall o d \in \mathcal{O D}, t_{s} \in \mathcal{M}, \tau \in \Gamma_{t_{s}}, t \in \mathcal{T} .
\end{aligned}
$$

where (24)-(26) are the KKT conditions and (27) is the market clearing condition.

After integrating the lower level complementarity constraints into the upper level problem, we obtain the equivalent one-level formulation of the multi-period bi-level MPEC formulation of the dynamic parking pricing and reservation problem:

$$
\begin{aligned}
& \max _{\mathbf{p}, \rho}\left\{\sum_{t=0}^{T} \gamma^{t} G_{t}\left(\mathbf{f}_{t}, \mathbf{p}_{t}\right) \mid \overline{\mathbf{f}}_{0}\right\} \\
& \text { s.t. }(1) \sim(6),(8),(24) \sim(27) .
\end{aligned}
$$

\section{Solution Methodology}

We propose to tackle the multi-period problem (28) using dynamic programming (DP) method. Using the DP terminology, we consider each decision epoch $t \in \mathcal{T}$ as a stage of dynamic system, and $\mathbf{f}_{t}$ as the state of system at stage $t$. Conservation constraints (5) and (6) are equivalent to the transition functions describing the system dynamics, and the objective function (8) can be regarded as the one-stage contribution function. The value function of state $\mathbf{f}_{t}$, denoting by $V_{t}\left(\mathbf{f}_{t}\right)$, should satisfy the following Bellman's optimality equation:

$$
V_{t}\left(\mathbf{f}_{t}\right)=\max _{\mathbf{p}_{t}}\left\{G_{t}\left(\mathbf{f}_{t}, \mathbf{p}_{t}\right)+\gamma V_{t+1}\left(\mathbf{f}_{t+1}\right)\right\}, \forall \mathbf{f}_{t}, \forall t .
$$

\footnotetext{
${ }^{2}$ Since $\lambda_{i}$ can either be interpreted as the shadow price of parking for user $i$, or the parking disutility of user $i$, and since $\lambda_{i}=\lambda_{i^{\prime}}, \forall i^{\prime} \in I_{t, o d, t_{s}, \tau}$, the equation $u=\lambda_{i}$ therefore holds.
} 
Typically, we solve (29) by means of backward recursion, in which $V_{t}\left(f_{t}\right)$ is computed from $V_{t+1}\left(f_{t+1}\right)$ by stepping backward in time. Thus, if $V_{t+1}(\cdot)$ is known for all possible states $\mathbf{f}_{t+1}$, an optimal pricing strategy $\mathbf{p}_{t}^{*}$ can be obtained by solving (29) in an exact way. Unfortunately, given the size of the state space and the embedded MPEC problem, exact computation of (29) is extremely difficult. Rather than applying backward recursion directly, we apply the ADP approach (Powell, 2011) to solve the problem in an approximate way. To see the need for this, we consider a very small instance (two parking lots, two parking spaces in each lot, a reservation horizon of two time periods, and parking price between $\$ 0$ and $\$ 2$ ). If we were to solve it via exact DP, we enforce all the state variables to be integer (such that the entire state space can be enumerated) and solve the Bellmans equation (29) exactly. The DP results after nearly an hour of computation show an optimal objective function value of 9.2. In contrast, the ADP approach, whose detail will be presented in this section, can converge within less than 30 seconds (200 iterations) to a good solution with an objective function value of 8.9. This small problem instance is already showing the significant computational benefit of ADP. If the problem size becomes even slightly larger, the state space would dramatically increase and the exact DP approach will no longer be a feasible option. For instance, for a small case with 5 parking lots, 10 parking spaces in each lot and 4 time periods in the reservation horizon, the state space is estimated to be $O\left(10^{30}\right)$. Even if we assume that the time for solving each Bellman's equation remains the same as that for the very small instance above, the total computation time would be at least $O\left(10^{26}\right)$ hours, which is unacceptable for real-world applications.

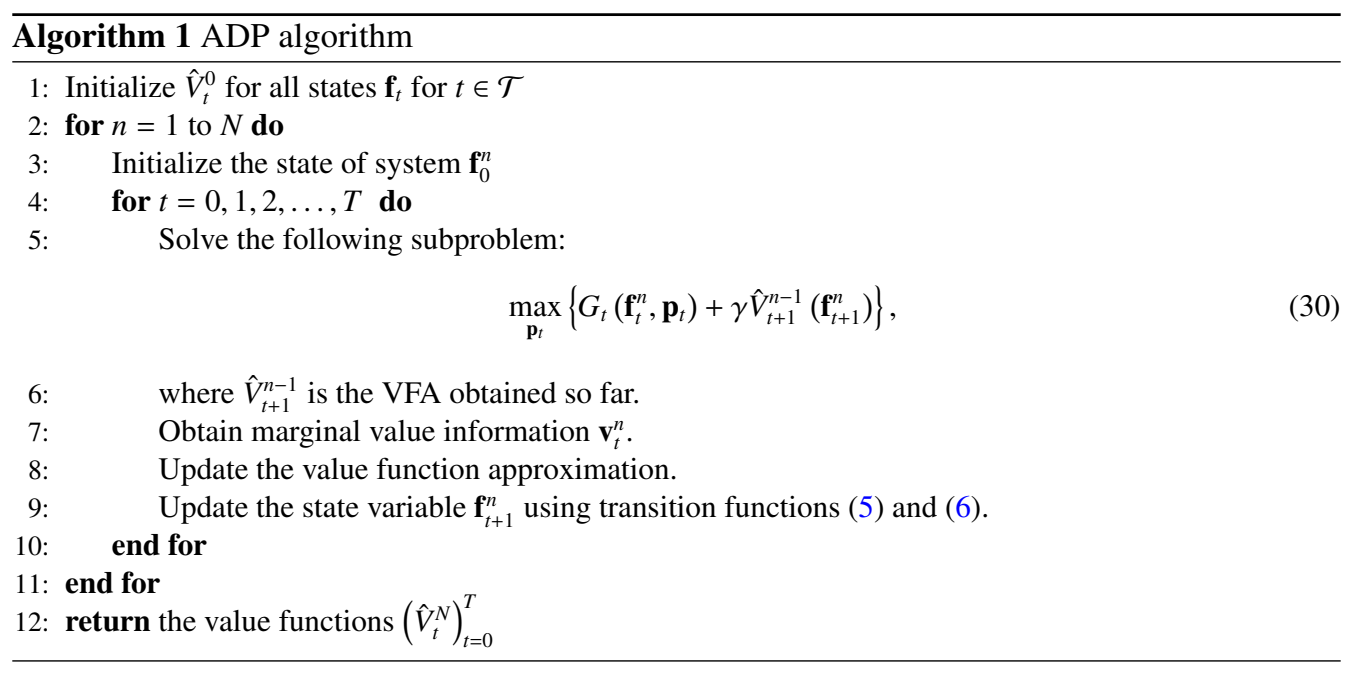

We present the basic framework of the ADP in pseudocode Algorithm 1 above. In general, the ADP approach consists of three key components: 1) a suitable designed value function approximation (VFA) $\left.\hat{V}_{t+1}\left(f_{t+1}\right) ; 2\right)$ a forward simulation by solving a sequence of one-stage subproblems (30); and 3) an update mechanism to improve the VFA in each iteration. We discuss the design of the VFA in Section 4.1, the update mechanism of the VFA in Section 4.2 and the solution approach to solve the one-stage subproblem (30) in Section 4.3. 


\subsection{Value function approximation}

In this work, the VFA is constructed as a concave separable piecewise linear function of state variables. For each $t \in \mathcal{T}$, we approximate $V_{t}\left(\mathbf{f}_{t}\right)$ by summing over a set of one-dimensional separable functions, i.e.,

$$
\hat{V}_{t}\left(\mathbf{f}_{t}\right)=\sum_{j} \sum_{m} \hat{V}_{t m j}\left(f_{t m j}\right)
$$

where $\hat{V}_{t m j}\left(f_{t m j}\right)$ is the concave piecewise linear function of $f_{t m j}$. We assume that the breakpoints of the piecewise linear function $\hat{V}_{t m j}\left(f_{t m j}\right)$ only occur at integer values of $f_{t m j}$. The piecewise linear function can provide a more stable approximation than a purely linear function, and the concave piecewise linear segments capture the decreasing marginal value of resources. Furthermore, a concave piecewise linear function can be easily embedded into an MIP or MIQP model with a maximization objective, such that the model can be solved using existing solvers.

\subsection{Updating value function approximation}

The marginal value information $\mathbf{v}_{t}^{n}$ mentioned in step 7 of Algorithm 1 refers to the benefit for having one additional unit of space at a given parking lot; i.e., the dual values associated with the capacity constraints $\sum_{\text {od }} \sum_{t_{s}=1}^{m} \sum_{\tau=m+1-t_{s}}^{M+1-t_{s}} h_{t, o d, t_{s}, \tau, j} \leq f_{t m j}, \forall j \in \mathcal{J}, m \in \mathcal{M}$, i.e., $\mathbf{v}_{t}=\left[\rho_{t m j}\right]_{\forall j \in \mathcal{J}, \forall m \in \mathcal{M}}$. A notable advantage of using $\rho_{t m j}$ is that its value can be obtained as a byproduct from solving (30) and thus additional computational efforts can be avoided.

After we have $\mathbf{v}_{t}^{n}$, the VFA can be updated by the following rules:

$$
\hat{V}_{t}^{n}(\mathbf{f})=\left\{\begin{array}{lr}
\left(1-\theta^{n-1}\right) \hat{V}_{t}^{n-1}(\mathbf{f})+\theta^{n-1} \mathbf{v}_{t}^{n}, & \text { for } \mathbf{f}=\mathbf{f}_{t}^{n}, \\
\hat{V}_{t}^{n-1}(\mathbf{f}), & \text { otherwise, }
\end{array}\right.
$$

where $\theta^{n-1} \in[0,1]$ is the step size. Here we use a simple harmonic step size rule $\theta^{n}=1 /(K+n)$, where $K$ is a positive integer constant.

From (31), we observe that only the VFA for state $\mathbf{f}=\mathbf{f}_{t}^{n}$ is updated. It is possible that the updated approximation no longer preserves concavity, i.e., $\hat{V}_{t}^{n}\left(\mathbf{f}_{t}^{n}\right)<\hat{V}_{t}^{n}\left(\mathbf{f}_{t}^{n}+1\right)$ or $\hat{V}_{t}^{n}\left(\mathbf{f}_{t}^{n}\right)>$ $\hat{V}_{t}^{n}\left(\mathbf{f}_{t}^{n}-1\right)$. In order to maintain the concavity of piecewise linear function $\hat{V}_{t m j}\left(f_{t m j}\right)$ at each iteration, we apply the SPAR algorithm of Powell et al. (2004) to smoothen the updated VFA. The fundamental idea of the SPAR algorithm is to average the values for $f^{n}$ to the left $\left(f^{n}-1\right)$ or right $\left(f^{n}+1\right)$ where a concavity violation exists, and keep moving toward to either direction until a monotonic concave function is attained.

\subsection{Reformulation of the one-stage subproblem}

Due to the nonconvex bilinear term in the objective function (8) and the inherent complexity in handling the complementarity constraints (24) (27), the problem (30) is computationally challenging in its current form. In the next, we first show how to transform the non-convex bilinear terms in (8) into equivalent convex quadratic and linear form based on the derivations in Hobbs et al. (2000) and Mackowski et al. (2015). 
By summing up (24) over $o d \in O \mathcal{D}, t_{s} \in \mathcal{M}, \tau \in \Gamma_{t_{s}}$ and $j \in \mathcal{J}$, we can have the following equation:

$$
\begin{aligned}
\sum_{o d} \sum_{t_{s}} \sum_{\tau} \sum_{j \in \mathcal{J}} \sum_{m=t_{s}}^{t_{s}+\tau-1} p_{t m j} h_{t, o d, t_{s}, \tau, j}=\sum_{o d} \sum_{t_{s}} \sum_{\tau} \sum_{j \in \mathcal{T}} h_{t, o d, t_{s}, \tau, j} u_{t, o d, t_{s}, \tau} \\
-\sum_{o d} \sum_{t_{s}} \sum_{\tau} \sum_{j \in \mathcal{J}}\left(\varphi_{o d, j}+\sum_{m \in \mathcal{M}_{t, s}^{\prime},} \rho_{t m j}\right) h_{t, o d, t_{s}, \tau, j}
\end{aligned}
$$

The first term on the right hand side of equation (32) can be derived as:

$$
\begin{aligned}
& \sum_{o d} \sum_{t_{s}} \sum_{\tau} \sum_{j \in \mathcal{T}} h_{t, o d, t_{s}, \tau, j} u_{t, o d, t_{s}, \tau} \\
& =\sum_{o d} \sum_{t_{s}} \sum_{\tau}\left(\sum_{j \in \mathcal{T} \cup \zeta} h_{t, o d, t_{s}, \tau, j}-h_{t, o d, t_{s}, \tau, \zeta}\right) u_{t, o d, t_{s}, \tau} \\
& =\sum_{o d} \sum_{t_{s}} \sum_{\tau} \sum_{j \in \mathcal{T} \cup \zeta} h_{t, o d, t_{s}, \tau, j} u_{t, o d, t_{s}, \tau}-\sum_{o d} \sum_{t_{s}} \sum_{\tau} h_{t, o d, t_{s}, \tau, \zeta} u_{t, o d, t_{s}, \tau} .
\end{aligned}
$$

Based on equation (4), we can have

$$
\sum_{o d} \sum_{t_{s}} \sum_{\tau} \sum_{j \in \mathcal{J} \cup \zeta} h_{t, o d, t_{s}, \tau, j} u_{t, o d, t_{s}, \tau}=\sum_{o d} \sum_{t_{s}} \sum_{\tau} H\left(u_{t, o d, t_{s}, \tau}\right) u_{t, o d, t_{s}, \tau} .
$$

Next, by summing up (25) over $o d \in O \mathcal{D}, t_{s} \in \mathcal{M}$ and $\tau \in \Gamma_{t_{s}}$, we can have

$$
\sum_{o d} \sum_{t_{s}} \sum_{\tau} h_{t, o d, t_{s}, \tau, \zeta} u_{t, o d, t_{s}, \tau}=\sum_{o d} \sum_{t_{s}} \sum_{\tau} \Phi_{t, o d, t_{s}, \tau, \zeta} h_{t, o d, t_{s}, \tau, \zeta} .
$$

Hence, equation (33) can be further derived as:

$$
\begin{aligned}
\sum_{o d} \sum_{t_{s}} \sum_{\tau} \sum_{j \in \mathcal{J}} h_{t, o d, t_{s}, \tau, j} u_{t, o d, t_{s}, \tau} & =\sum_{o d} \sum_{t_{s}} \sum_{\tau} H\left(u_{t, o d, t_{s}, \tau}\right) \\
& u_{t, o d, t_{s}, \tau} \\
& -\sum_{o d} \sum_{t_{s}} \sum_{\tau} \Phi_{t, o d, t_{s}, \tau, \zeta} h_{t, o d, t_{s}, \tau, \zeta} .
\end{aligned}
$$

Given the assumption that $H(u)=a-b u$, it is easy to know that the right hand side of equation (34) only contains linear and quadratic terms. The second term on the right hand side of equation (32) can be derived as:

$$
\begin{aligned}
\sum_{o d} \sum_{t_{s}} \sum_{\tau} \sum_{j \in \mathcal{J}}\left(\varphi_{o d, j}\right. & \left.+\sum_{m \in \mathcal{M}_{s, \tau}^{\prime}} \rho_{t m j}\right) h_{t, o d, t_{s}, \tau, j} \\
& =\sum_{o d} \sum_{t_{s}} \sum_{\tau} \sum_{j \in \mathcal{J}} \varphi_{o d, j} h_{t, o d, t_{s}, \tau, j}+\sum_{o d} \sum_{j \in \mathcal{J}} \sum_{t_{s}} \sum_{\tau} \sum_{m \in \mathcal{M}_{t s, \tau}^{\prime}} \rho_{t m j} h_{t, o d, t_{s}, \tau, j} .
\end{aligned}
$$

By summing up (26) over $m \in \mathcal{M}$ and $j \in \mathcal{J}$, we can have

$$
\sum_{o d} \sum_{j \in \mathcal{T}} \sum_{m} \sum_{t_{s}=1}^{m} \sum_{\tau=m+1-t_{s}}^{M+1-t_{s}} \rho_{t m j} h_{t, o d, t_{s}, \tau, j}=\sum_{j \in \mathcal{J}} \sum_{m} f_{t m j} \rho_{t m j} .
$$


Proposition 1. The following equation (36) holds.

$$
\sum_{o d} \sum_{j \in \mathcal{J}} \sum_{t_{s}} \sum_{\tau} \sum_{m \in \mathcal{M}_{t, \tau}^{\prime}, \tau} \rho_{t m j} h_{t, o d, t_{s}, \tau, j}=\sum_{o d} \sum_{j \in \mathcal{J}} \sum_{m} \sum_{t_{s}=1}^{m} \sum_{\tau=m+1-t_{s}}^{M+1-t_{s}} \rho_{t m j} h_{t, o d, t_{s}, \tau, j} .
$$

Proof. See Appendix A. $\square$

Combining the result of Proposition 1, (35) can be derived as:

$$
\begin{aligned}
\sum_{o d} \sum_{t_{s}} \sum_{\tau} \sum_{j \in \mathcal{J}}\left(\varphi_{o d, j}+\sum_{m \in \mathcal{M}_{t s, \tau}^{\prime}} \rho_{t m j}\right) h_{t, o d, t_{s}, \tau, j}=\sum_{o d} \sum_{t_{s}} \sum_{\tau} \sum_{j \in \mathcal{T}} \varphi_{o d, j} h_{t, o d, t_{s}, \tau, j} & +\sum_{j \in \mathcal{J}} \sum_{m} f_{t m j} \rho_{t m j} .
\end{aligned}
$$

Integrating (32), (34) and (37), we are able to reformulate the bilinear term $\sum_{o d} \sum_{t_{s}} \sum_{\tau} \sum_{j} \sum_{m} p_{t m j} h_{t, o d, t_{s}, \tau, j}$ into the following linear and quadratic form:

$$
\begin{aligned}
\sum_{o d} \sum_{t_{s}} \sum_{\tau} \sum_{j \in \mathcal{J}} \sum_{m=t_{s}}^{t_{s}+\tau-1} p_{t m j} h_{t, o d, t_{s}, \tau, j}= & \sum_{o d} \sum_{t_{s}} \sum_{\tau} H\left(u_{t, o d, t_{s}, \tau}\right) u_{t, o d, t_{s}, \tau}-\sum_{o d} \sum_{t_{s}} \sum_{\tau} \Phi_{t, o d, t_{s}, \tau, \zeta} h_{t, o d, t_{s}, \tau, \zeta} \\
& -\sum_{o d} \sum_{t_{s}} \sum_{\tau} \sum_{j \in \mathcal{T}} \varphi_{o d, j} h_{t, o d, t_{s}, \tau, j}-\sum_{j \in \mathcal{T}} \sum_{m} f_{t m j} \rho_{t m j} .
\end{aligned}
$$

Similarly, another bilinear term $\sum_{o d} \sum_{t_{s}} \sum_{\tau} \sum_{j} \sum_{m} h_{t, o d, t_{s}, \tau, j} u_{t, o d, t_{s}, \tau}$ can be reformulated as:

$$
\begin{aligned}
\sum_{o d} \sum_{t_{s}} \sum_{\tau} \sum_{j \in \mathcal{J}} \sum_{m=t_{s}}^{t_{s}+\tau-1} h_{t, o d, t_{s}, \tau, j} u_{t, o d, t_{s}, \tau}=\sum_{o d} \sum_{t_{s}} \sum_{\tau} H\left(u_{t, o d, t_{s}, \tau}\right) u_{t, o d, t_{s}, \tau} & -\sum_{o d} \sum_{t_{s}} \sum_{\tau} \Phi_{t, o d, t_{s}, \tau, \zeta} h_{t, o d, t_{s}, \tau, \zeta} .
\end{aligned}
$$

The reformulated quadratic and linear terms can be proven to be convex based on Bai et al. (2012b) and Hobbs et al. (2000).

We reformulate the complementarity constraints (24)-(26) by incorporating disjunctive constraints. ${ }^{3}$ The underlying rationale is to decouple the complementarity constraints into multiple mixed-integer linear constraints by introducing an additional indicator variable for each complementarity constraint (Gabriel and Leuthold, 2010; Bai et al., 2012b, 2016). First, we define $q_{t, o d, t_{s}, \tau, m, j} \in\{0,1\}, s_{t, o d, t_{s}, \tau, \zeta} \in\{0,1\}$ and $r_{t m j} \in\{0,1\}$ as indicator variables associated with constraints (24)-(26), respectively. Then, we attempt to derive the valid bounds for both sides of

\footnotetext{
${ }^{3}$ Note that if the problem is directly solved by applying a nonlinear program solver, certain procedures (e.g., the quadratic penalty method) should be followed to make sure that the complementarity constraints satisfy the constraint qualification (Izmailov and Solodov, 2004; Rodrigues and Monteiro, 2006; Friesz et al., 2013).
} 
the complementarity constraints. The upper bound and lower bound for $u_{t, o d, t_{s}, \tau}$ are respectively defined as follows:

$$
\begin{aligned}
& \bar{u}_{t, o d, t_{s}, \tau}^{\mathrm{UB}}=\min \left\{Y^{-1}(0), \max _{j \in \mathcal{J}}\left\{\tau P_{j}^{U}+\varphi_{o d, j}\right\}\right\}, \quad \forall o d \in O \mathcal{D}, t_{s} \in \mathcal{M}, \tau \in \Gamma_{t_{s}}, \\
& \bar{u}_{t, o d, t_{s}, \tau}^{\mathrm{LB}}=\min _{j}\left\{\tau P_{j}^{L}+\varphi_{o d, j}\right\}, \quad \forall o d \in O \mathcal{D}, t_{s} \in \mathcal{M}, \tau \in \Gamma_{t_{s} .}
\end{aligned}
$$

The upper bound of the $h_{t, o d, t_{s}, \tau, j}$ can be defined as:

$$
\bar{h}_{t, o d, t, t, \tau, j}^{\mathrm{UB}}=\min \left\{\min _{m \in \mathcal{M}_{t_{s}, \tau}^{\prime}}\left\{f_{t m j}\right\}, H\left(\bar{u}_{t, o d, t_{s}, \tau}^{\mathrm{LB}}\right)\right\}, \quad \forall o d \in \mathcal{O D}, t_{s} \in \mathcal{M}, \tau \in \Gamma_{t_{s}}, j \in \mathcal{J} .
$$

Similarly, the upper bound of the $h_{t, o d, t_{s}, \tau, \zeta}$ can be defined as:

$$
\bar{h}_{t, o d, t_{s}, \tau, \zeta}^{\mathrm{UB}}=H\left(\bar{u}_{t, o d, t_{s}, \tau}^{\mathrm{LB}}\right), \quad \forall o d \in O \mathcal{D}, t_{s} \in \mathcal{M}, \tau \in \Gamma_{t_{s}} .
$$

Proposition 2. A valid upper bound to $\rho_{t m j}, \forall j \in \mathcal{J}, \forall m \in \mathcal{M}$, is given as follows

$$
\bar{\rho}_{t m j}^{U B}=\max _{\substack{\forall o d, y t_{s} \in\{1, \ldots, m\}, m+1-t_{s} \leq \tau \leq M+1-t_{s}}}\left\{\bar{u}_{t, o d, t_{s}, \tau}^{U B}-\bar{u}_{t, o d, t_{s}, \tau}^{L B}\right\} .
$$

Proof. See Appendix A. $\square$

Therefore, the complementarity constraints (24)-(26) can be reformulated as follows:

$$
\begin{aligned}
& 0 \leq h_{t, o d, t_{s}, \tau, j} \leq \bar{h}_{t, o d, t_{s}, \tau, j}^{\mathrm{UB}} q_{t, o d, t_{s}, \tau, j}, \quad \forall o d \in O \mathcal{D}, t_{s} \in \mathcal{M}, \tau \in \Gamma_{t_{s}}, j \in \mathcal{J}, \\
& 0 \leq \phi_{t, o d, t_{s}, \tau, j}+\sum_{m \in \mathcal{M}_{t, \tau}^{\prime}, \tau} \rho_{t m j}-u_{t, o d, t_{s}, \tau} \leq\left(\bar{\phi}_{t, o d, t_{s}, \tau, j}^{\mathrm{UB}}+\sum_{m \in \mathcal{M}_{t, \tau}^{\prime}, \tau} \bar{\rho}_{t m j}^{\mathrm{UB}}-\bar{u}_{t, o d, t_{s}, \tau}^{\mathrm{LB}}\right)\left(1-q_{t, o d, t_{s}, \tau, j}\right), \\
& \forall o d \in O \mathcal{D}, t_{s} \in \mathcal{M}, \tau \in \Gamma_{t_{s}}, j \in \mathcal{J}, \\
& 0 \leq \Phi_{t, o d, t_{s}, \tau, \zeta}-u_{t, o d, t_{s}, \tau} \leq\left(\Phi_{t, o d, t_{s}, \tau, \zeta}-\bar{u}_{t, o d, t_{s}, \tau}^{\mathrm{LB}}\right)\left(1-s_{t, o d, t_{s}, \tau, \zeta}\right), \\
& \forall o d \in O \mathcal{D}, t_{s} \in \mathcal{M}, \tau \in \Gamma_{t_{s}}, \\
& 0 \leq h_{t, o d, t_{s}, \tau, \zeta} \leq \bar{h}_{t, o d, t_{s}, \tau, \zeta}^{\mathrm{UB}} s_{t, o d, t_{s}, \tau, \zeta}, \quad \forall o d \in O \mathcal{D}, t_{s} \in \mathcal{M}, \tau \in \Gamma_{t_{s}}, \\
& 0 \leq \rho_{t m j} \leq \bar{\rho}_{t m j}^{\mathrm{UB}} r_{t m j}, \quad \forall j \in \mathcal{J}, m \in \mathcal{M} \\
& 0 \leq f_{t m j}-\sum_{o d} \sum_{t_{s}=1}^{m} \sum_{\tau=m+1-t_{s}}^{M+1-t_{s}} h_{t, o d, t_{s}, \tau, j} \leq f_{t m j}\left(1-r_{t m j}\right), \quad \forall j \in \mathcal{J}, m \in \mathcal{M}
\end{aligned}
$$

To this end, we can present the reformulated version of the one-stage model at stage $t \in \mathcal{T}$ as follows:

$$
\begin{array}{r}
\max _{\mathbf{p}_{t}}\left\{G_{t}\left(\mathbf{f}_{t}, \mathbf{p}_{t}\right)+\gamma \hat{V}_{t+1}\left(\mathbf{f}_{t+1}\right)\right\} \\
\text { s.t. (1) } \sim(6),(8),(38) \sim(45)
\end{array}
$$

We can see that the original one-stage model at each stage has been successfully transformed into an equivalent single-level concave MIQP, which can be solved using the off-the-shelf MIQP solvers (e.g., CPLEX). 


\section{Numerical Experiments}

In this section, we present a series of numerical experiments to test the performance of the proposed model and the ADP algorithm. We consider a test case with 15 university-owned parking lots (with a total capacity of 295 parking spaces) on the campus of the University of Illinois at Urbana-Champaign. The agency updates location-dependent parking prices every 30 minutes within a six-hour time horizon. At each update time, a three-hour reservation horizon (containing 6 periods) is considered.

(Place Figure 3 about here).

The geographic distribution of the 15 parking lots is depicted as rectangle boxes in Figure 3 . Commuters arrive on campus from either the west or the east campus access point (shown as arrows) and head toward one of three major attraction zones (shown as circles). The driving time from the access points to the parking lots as well as the walking time from parking lots to destinations are computed in advance. The monetary value of walking time is assumed to be $\$ 0.28 / \mathrm{min}$ (80\% of the average hourly wage in Champaign-Urbana area, i.e., $\$ 21.56$ (Bureau of Labor Statistics, 2014)) and that of driving time $\$ 0.18 / \mathrm{min}$ (50\% of the average hourly wage); the value of $\varphi_{t, o d, j}$ can be calculated. The parking price fluctuation range is set as $\epsilon_{l}=\epsilon_{r}=\$ 0.5$, whereas no maximum and minimum price limits are imposed in this work, i.e., $P_{j}^{L}=0$ and $P_{j}^{U}=\infty, \forall j \in \mathcal{J}$.

Follow the demand configuration approach in Mackowski et al. (2015), we use a multi-modal distribution (46) to approximate the linear demand curve's intercept for each type of users,

$$
a_{t, o d, t_{s}, \tau}=k \pi_{o d} v_{\tau}\left(\sum_{t_{p}=1}^{3} \frac{\Omega_{t_{p}}}{\sqrt{\sigma_{t_{p}}}} e^{-\left(\frac{t+t_{s}-\mu_{t_{p}}}{\sigma_{t_{p}}}\right)^{2}}\right),
$$

where $k$ denotes the percentage of vehicle trips that use the parking reservation system, $\pi_{o d}$ denote the percentage of vehicle trips associated with each OD pair, $v_{\tau}$ denotes the fraction of trips with parking duration $\tau, \Omega_{t_{p}}$ represents the total number of trips to the studied area during time-ofday period $t_{p}, \sigma_{t_{p}}$ is the duration of $t_{p}$, and $\mu_{t_{p}}$ denotes the middle time of $t_{p}$. In the following tests, unless explicitly stated, demand $\Omega_{t_{p}}$ is an increasing function of $t_{p}$. We consider three different times-of-day periods. We assume all types of users share the same demand elasticity in the following numerical tests, i.e., $b_{t, o d, t_{s}, \tau}=-0.3, \forall t, o d, t_{s}, \tau$ (TCRP, 2005). ${ }^{4}$ The MIQP at each stage is solved using the commercial solver CPLEX, and the ADP algorithm goes through 20 iterations every time it is called upon. All tests are performed on a personal computer with 3.4GHz CPU and 8GB RAM.

Table 1 shows results for eleven test cases, each with a different set of weights for economic surplus and occupancy imbalance penalty (i.e., $\alpha_{1}$ and $\alpha_{2}$ respectively). For each test case, we apply the ADP algorithm first to obtain the corresponding VFA, and then evaluate both the myopic policy and the ADP policy under the same simulation settings. As in Mackowski et al. (2015), the myopic policy is to solve an MIQP problem at each stage without considering the VFA, i.e., at each stage $t$, the pricing decision is obtained by solving an optimization problem as

\footnotetext{
${ }^{4}$ The parking demand elasticity for a certain urban area could be calibrated through field experiments, i.e., by adjusting the parking price and then observing how the demand would change (Pierce and Shoup, 2013).
} 
follows:

$$
\begin{aligned}
\max _{\mathbf{p}_{t}} & G_{t}\left(\mathbf{f}_{t}, \mathbf{p}_{t}\right) \\
\text { s.t. } & (1) \sim(6),(8),(38) \sim(45)
\end{aligned}
$$

As we can see that in the myopic pricing policy, no future information is considered in the current decision-making process, and the possible impacts of the current decision on the future states are simply ignored.

(Place Table 1 and Table 2 about here).

Table 1 also presents the computation time to run the ADP algorithm and the total objective function values under myopic and ADP pricing policies, respectively. We can see that the average computation time of a single ADP iteration (i.e., solving the MIQP subproblem) is no longer than $10 \mathrm{~min}$. The solution efficiency is acceptable in real life. From columns 5 to 7 , it is easy to observe that the ADP-based pricing policy consistently outperforms the myopic counterpart in terms of the total objective function value. In Table 2 , we present the results of economic surplus and occupancy imbalance penalty under both policies. Note that these potential objectives are both improved by applying the ADP policy.

(Place Figure 4 about here).

The average parking prices as functions of time from the two solution approaches are illustrated in Figure 4. Note that the parking price for a given lot and a given time period may vary across decision epochs. In the figure, each shown price (for a time period) is averaged across both decision epochs and across parking lots.

The myopic pricing policy tends to offer a low price at the initial stage to attract more demand. However, as the demand rapidly increases as time passes, the myopic policy seems to be unable to change the prices accordingly. The main reason is that, due to the previous reservations at low prices, most of the parking spaces have been already booked and the system has lost the ability to accept newly coming demand in the later periods. As we all know, the influence of price over demand is quite limited if there are no products to supply. Hence, there is no need to change the parking prices if the system has already run out of spaces. To the contrary, we can observe that the ADP pricing policy inclines to set a relatively higher price at the early stage of the planning horizon, such that some of the parking spaces can be saved up for more profitable reservations in the near future. Moreover, the ADP policy changes the prices in a more sensitive way so as to provide quicker responses to demand variations throughout the planning horizon.

To find out the source of improvement for the ADP policy, we further investigate how revenue, consumer surplus, and occupancy imbalance objectives are individually affected by two different approaches. Here we use the case $\left(\alpha_{1}=0.5, \alpha_{2}=0.5\right)$ to run the test, and the relevant results are displayed in Figure 5. As shown in Figure 5a, the ability to generate revenue at the beginning part of the horizon are similar to both policies. As time passes, the revenue obtained from the myopic approach suffers from a sharp decrease, while the revenue from the ADP approach has a significant increase. Similar patterns are also observed for the consumer surplus, the occupancy imbalance penalty, and the total objective function values (see Figure $5 \mathrm{~b}$ $5 \mathrm{~d}$ ). Therefore, by setting relatively higher prices before the arrival of huge demand, the ADP policy successfully improves the system performance.

(Place Figure 5 about here). 


\subsection{Effect of consumer surplus}

In this section, we run tests to demonstrate the importance of incorporating consumer surplus term,

$$
\sum_{o d} \sum_{t_{s}} \sum_{\tau} \sum_{j} \frac{1}{2} h_{t, o d, t_{s}, \tau, j}\left(u_{t, o d, t_{s}, \tau}^{\max }-u_{t, o d, t_{s}, \tau}\right),
$$

into the agency's objective. First, we create a benchmark scenario by excluding the consumer surplus term, and then solve both models by the ADP algorithm under the same parameter settings. Here we perform the tests using test case $\left(\alpha_{1}=1, \alpha_{2}=0\right)$ to eliminate the influence of the occupancy balance objective over the solutions. After we obtain the pricing decision, the values of consumer surplus are computed according to (47). The comparison is summarized in Table 3 , and the parking prices for both scenarios are shown in Figure 6.

(Place Table 3 and Figure 6 about here).

Even though the parking prices and the associated revenue are relatively lower, the inclusion of consumer surplus leads to a greater total objective function value. The rationale behind is that the consumer surplus term in the objective function inclines to push down the parking prices to increase the consumer surplus, such that extremely high parking prices can be prevented. Therefore, unless the agency runs a profit-centered business, it is necessary to include the consumer surplus if the agency wants to increase the satisfaction level of the customers by imposing a more affordable price for the parking system.

\subsection{Effect of different demand patterns}

The above experiments are all conducted for increasing demand over time. In the following, we attempt to investigate the robustness of the ADP pricing policy for different demand patterns. Other than the increasing demand assumed earlier, a bell-shaped and a decreasing demand patterns are considered. The shape of demand patterns and the results of parking prices are displayed in Figure 4, 7 and 8, respectively. The associated results of the objective function values are summarized in Table 4.

(Place Table 4, Figure 7 and Figure 8 about here).

From Table 4, we can observe that the objective function values from the ADP policy are better for all three cases. In particular, the ADP policy shows a significant advantage in handling the increasing and bell-shaped demand patterns. However, it should be noted that the advantage of ADP policy is much less obvious for the decreasing demand pattern. This is intuitive. As shown in Figure 8, the myopic policy would also raise up the price at early stages of the planning horizon to deal with the immediate high level of demand. Meanwhile, since the demand will gradually decrease as the time passing, the motivation to save up parking spaces for future use is less pressing. Therefore, the performance of the myopic pricing policy is generally similar to the ADP approach for the decreasing demand pattern.

\subsection{Effect of spatial and temporal disparity in parking demand}

In the following, we test the performance of the ADP pricing policy against the myopic counterpart by allowing different demand destinations to have different demand patterns.

For simplicity of illustration, we focus on two demand attraction areas (D1 and D3) in the following tests. In order to eliminate the potential interference of D-2, we assuming that there is no demand going toward to D-2 and the parking lots close to D-2 but far from D-1 and D-3 (i.e., P-6 and P-7) are excluded from the system. The remaining parking lots are then categorized into 
three classes: i) the ones close to D-1 but far from D-3 (P-1 P-5), ii) the ones close to D-3 but far from D-1 (P-11 P-15), iii) the ones close to both areas (P-8 P-10). The demand patterns for D-1 and D-3 are shown in Figure 9a. As we can see, the peak of demand for D-3 occurs at an early time of the planning horizon while the demand peak for D-1 occurs at a later time. The parking prices for each class of parking lots are shown in Figure 9b 9d.

(Place Figure 9 about here).

As more demand goes toward to D-3 at an early time, the myopic policy tends to set relatively higher prices (e.g., \$0.4 at period 3) for the parking lots close to D-3 (as shown in Figure 9c) and lower prices (e.g., \$0.2 at period 3) for the others (as shown in Figure 9b and 9d). However, the ADP policy sets higher prices (e.g., \$0.7 at period 3) for the parking lots around D-1 early on (as shown in Figure 9b), especially for those parking lots close to D-3 (as shown in Figure 9d). The reason is that, if we can take account of the future demand information into the earlier decisionmaking process, it appears to be more beneficial to save up some convenient parking spaces near D-1 for more valuable use in the future. The results in Table 5 clearly show that the ADP policy outperforms the myopic one with respect to all performance measure, indicating that the ADP approach is able to effectively handle both spatial and temporal disparity in parking demand.

(Place Table 5 about here).

\section{Conclusion}

This paper studies a demand-driven parking pricing and reservation problem, in which the agency decides the spatial and temporal distribution of parking prices so as to achieve a systemwide objective, while drivers with different origins and destinations compete for limited parking spaces through online reservations. The proposed dynamic Stackelberg leader-follower game is formulated as a multi-period non-cooperative bi-level MPEC model, in which the agency makes pricing decision in the upper level and the drivers make parking location decisions in the lower level. A non-myopic ADP algorithm is developed to solve the proposed model. We further design an embedded subroutines to transform the bi-level MPEC model in each time period into a solvable single-level MIQP. A test case based on the campus of the University of Illinois at Urbana-Champaign is constructed to test the performance of the proposed model and algorithm. Through extensive numerical experiments, we show that the ADP-based pricing policy is able to achieve better system performance, especially by incorporating future parking supply and demand information into dynamic pricing decisions. The ADP approach also exhibits robustness in dealing with both spatial and temporal variations in parking demand.

There are many possible future research opportunities. In our research, all the parameters are assumed as deterministic, whereas in reality uncertainty widely exists in demand and user behavior. In that case, the pricing policy of the deterministic model may become inappropriate and even result in inferior system performance. Hence, gaming behavior between the agency and the drivers under uncertainty is an interesting topic for future research. In addition, future efforts can be devoted to exploring more complex parking behaviors of drivers. For example, the drivers may adjust the time of their reservation or departure (delay or advance) to evade the high price periods. The choice of parking duration may also subject to change in according to the parking prices. Furthermore, we are also interested in extending out research by considering the engagement of multiple parking management agencies in pricing competition with each other. In this case, an equilibrium problem with equilibrium constraints (EPEC) is likely to be formulated in a dynamic setting. These possibilities could be considered in future studies. 


\section{Acknowledgments}

This research was supported in part by the U.S. National Science Foundation via Grant CMMI-1234085 and the Department of Civil and Environmental Engineering at the University of Illinois at Urbana-Champaign via a departmental Innovation Research Grant.

\section{Appendix A. Proof of propositions in Section 4.3}

Proposition 1. The following equation holds.

$$
\sum_{o d} \sum_{j \in \mathcal{J}} \sum_{t_{s}} \sum_{\tau} \sum_{m \in \mathcal{M}_{t s, \tau}^{\prime}} \rho_{t m j} h_{t, o d, t_{s}, \tau, j}=\sum_{o d} \sum_{j \in \mathcal{J}} \sum_{m} \sum_{t_{s}=1}^{m} \sum_{\tau=m+1-t_{s}}^{M+1-t_{s}} \rho_{t m j} h_{t, o d, t_{s}, \tau, j} .
$$

Proof. For simplicity of illustration, we omit the subscripts $t, j$ and $o d$ in the proof. (A.1) can thus be simplified as

$$
\sum_{t_{s}} \sum_{\tau} \sum_{m \in \mathcal{M}_{t s, \tau}^{\prime}} \rho_{m} h_{t_{s}, \tau}=\sum_{m} \sum_{t_{s}=1}^{m} \sum_{\tau=m+1-t_{s}}^{M+1-t_{s}} \rho_{m} h_{t_{s}, \tau}
$$

(Place Figure A.10 about here).

We take a small example (as shown in Figure A.10) to illustrate why equation (A.2) holds. The reservation horizon has four time periods. We display each positive $h$ as a rectangular box in Figure A.10. The rectangular boxes can be further divided into multiple smaller boxes for each time period. The small boxes are denoted as $\tilde{h}$ and indexed by the number shown in the box. For instance, $h_{t_{s}=1, \tau=2}$ actually consists of box 1 and 2 , such that $h_{t_{s}=1, \tau=2}=\tilde{h}_{1}+\tilde{h}_{2}$. The left side of (A.2) can be interpreted as the summation horizontally first and then vertically across all boxes, i.e.,

$$
\sum_{t_{s}} \sum_{\tau} \sum_{m \in \mathcal{M}_{t, \tau}^{\prime},} \rho_{m} h_{t_{s}, \tau}=\left[\left(\rho_{1} \tilde{h}_{1}+\rho_{2} \tilde{h}_{2}\right)+\left(\rho_{2} \tilde{h}_{3}+\rho_{3} \tilde{h}_{4}\right)+\left(\rho_{1} \tilde{h}_{5}+\rho_{2} \tilde{h}_{6}+\rho_{3} \tilde{h}_{7}\right)\right] .
$$

And the right side of (A.2) can be considered as summation vertically first and then horizontally across all boxes, i.e.,

$$
\sum_{m} \sum_{t_{s}=1}^{m} \sum_{\tau=m+1-t_{s}}^{M+1-t_{s}} \rho_{m} h_{t_{s}, \tau}=\left[\left(\rho_{1} \tilde{h}_{1}+\rho_{1} \tilde{h}_{5}\right)+\left(\rho_{2} \tilde{h}_{2}+\rho_{2} \tilde{h}_{3}+\rho_{2} \tilde{h}_{6}\right)+\left(\rho_{3} \tilde{h}_{4}+\rho_{3} \tilde{h}_{7}\right)\right] .
$$

It is easy to observe that (A.1) holds for this small example. The proposition is a simple generalization of the above example.

Proposition 2. A valid upper bound to $\rho_{t m j}, \forall j \in \mathcal{J}, \forall m \in \mathcal{M}$, is given as follows

$$
\bar{\rho}_{t m j}^{U B}=\max _{\substack{\forall o d, \forall t_{s} \in\{1, \ldots, m\}, m+1-t_{s} \leq \tau \leq M+1-t_{s}}}\left\{\bar{u}_{t, o d, t_{s}, \tau}^{U B}-\bar{u}_{t, o d, t_{s}, \tau}^{L B}\right\} .
$$


Proof. According to (24), we know that

$$
\phi_{t, o d, t_{s}, \tau, j}+\sum_{m \in \mathcal{M}_{t, \tau}^{\prime}} \rho_{t m j}-u_{t, o d, t_{s}, \tau} \geq 0, \quad \forall o d \in O \mathcal{D}, t_{s} \in \mathcal{M}, \tau \in \Gamma_{t_{s}}, j \in \mathcal{J}, t \in \mathcal{T},
$$

and as such, we could have

$$
\sum_{m \in \mathcal{M}_{t, \tau}^{\prime},} \rho_{t m j} \geq \phi_{t, o d, t_{s}, \tau, j}-u_{t, o d, t_{s}, \tau}, \quad \forall o d \in \mathcal{O D}, t_{s} \in \mathcal{M}, \tau \in \Gamma_{t_{s}}, j \in \mathcal{J}, t \in \mathcal{T}
$$

As we have already obtained the upper bound of $\phi_{t, o d, t_{s}, \tau, j}$ and the lower bound of $u_{t, o d, t_{s}, \tau}$, it is easy to know that the largest possible value for the right side of (A.4) is no greater than $\left(\bar{u}_{t, o d, t_{s}, \tau}^{\mathrm{UB}}-\bar{u}_{t, o d, t_{s}, \tau}^{\mathrm{LB}}\right)$. Note that there is no explicit upper bound restriction over the term $\sum_{m \in \mathcal{M}_{t, \tau}^{\prime}} \rho_{t m j}$ throughout the entire model. Hence, we can actually add an artificial upper bound for this term as long as it does not violate (A.4). It means that this upper bound should be no less than the largest possible value of the right-hand side of (A.4), i.e., $\left(\bar{u}_{t, o d, t_{s}, \tau}^{\mathrm{UB}}-\bar{u}_{t, o d, t_{s}, \tau}^{\mathrm{LB}}\right)$. Therefore, the upper bound of $\sum_{m \in \mathcal{M}_{t_{s}, \tau}^{\prime}} \rho_{t m j}$ can be any value that is larger than or equal to $\left(\bar{u}_{t, o d, t_{s}, \tau}^{\mathrm{UB}}-\bar{u}_{t, o d, t_{s}, \tau}^{\mathrm{LB}}\right)$. For computational simplicity, here we choose the smallest value to be the upper bound, i.e.,

$$
\sum_{m \in \mathcal{M}_{t, \tau}^{\prime},} \rho_{t m j} \leq\left(\bar{u}_{t, o d, t_{s}, \tau}^{\mathrm{UB}}-\bar{u}_{t, o d, t_{s}, \tau}^{\mathrm{LB}}\right) .
$$

The proposition can be proved according to the following inequalities:

$$
\rho_{t m j} \leq \sum_{m \in \mathcal{M}_{t, \tau}^{\prime}} \rho_{t m j} \leq\left(\bar{u}_{t, o d, t_{s}, \tau}^{\mathrm{UB}}-\bar{u}_{t, o d, t_{s}, \tau}^{\mathrm{LB}}\right) \leq \max _{\substack{\forall o d, \forall t_{s} \in\{1, \ldots, m\}, m+1-t_{s} \leq \tau \leq M+1-t_{s}}}\left\{\bar{u}_{t, o d, t_{s}, \tau}^{\mathrm{UB}}-\bar{u}_{t, o d, t_{s}, \tau}^{\mathrm{LB}}\right\},
$$

for $\forall o d \in O \mathcal{D}, t_{s} \in \mathcal{M}, \tau \in \Gamma_{t_{s}}, j \in \mathcal{J}, t \in \mathcal{T}$.

\section{References}

Arnott, R., de Palma, A., Lindsey, R., 1991. A temporal and spatial equilibrium analysis of commuter parking. Journal of Public Economics 45, 301-335.

Ayala, D., Wolfson, O., Xu, B., Dasgupta, B., Lin, J., 2011. Parking Slot Assignment Games, in: Proceedings of the 19th ACM SIGSPATIAL International Conference on Advances in Geographic Information Systems, ACM, New York, NY, USA. pp. 299-308.

Ayala, D., Wolfson, O., Xu, B., DasGupta, B., Lin, J., 2012. Pricing of Parking for Congestion Reduction, in: Proceedings of the 20th International Conference on Advances in Geographic Information Systems, ACM, New York, NY, USA pp. 43-51.

Bai, L., Mitchell, J.E., Pang, J.S., 2012a. On convex quadratic programs with linear complementarity constraints. Computational Optimization and Applications 54, 517-554.

Bai, Y., Ouyang, Y., Pang, J.S., 2012b. Biofuel supply chain design under competitive agricultural land use and feedstock market equilibrium. Energy Economics 34, 1623-1633.

Bai, Y., Ouyang, Y., Pang, J.S., 2016. Enhanced models and improved solution for competitive biofuel supply chain design under land use constraints. European Journal of Operational Research 249, 281-297.

Bertsekas, D.P., Tsitsiklis, J.N., 1996. Neuro-Dynamic Programming. 1 edition ed., Athena Scientific, Belmont, Mass.

Bureau of Labor Statistics, 2014. May 2014 metropolitan and nonmetropolitan area occupational employment and wage estimates in Champaign-Urbana, IL. http://www.bls.gov/oes/current/oes_16580.htm. Accessed: $01 / 10 / 2016$

Chen, Z., Xu, Z., Zangui, M., Yin, Y., 2016. Analysis of Advanced Management of Curbside Parking. Transportation Research Record: Journal of the Transportation Research Board 2567, 57-66. 
Chen, Z., Yin, Y., He, F., Lin, J.L., 2015. Parking Reservation for Managing Downtown Curbside Parking. Transportation Research Record: Journal of the Transportation Research Board 2498, 12-18.

Facchinei, F., Pang, J.S. (Eds.), 2004. Finite-Dimensional Variational Inequalities and Complementarity Problems. Springer Series in Operations Research and Financial Engineering, Springer New York, New York, NY.

Friesz, T.L., Han, K., Liu, H., Yao, T., 2013. Dynamic Congestion and Tolls with Mobile Source Emission. Procedia Social and Behavioral Sciences 80, 818-836.

Gabriel, S.A., Leuthold, F.U., 2010. Solving discretely-constrained MPEC problems with applications in electric power markets. Energy Economics 32, 3-14.

Geng, Y., Cassandras, C., 2011. Dynamic resource allocation in urban settings: A smart parking approach, in: 2011 IEEE International Symposium on Computer-Aided Control System Design (CACSD), pp. 1-6.

Guo, L., Huang, S., Zhuang, J., Sadek, A.W., 2012. Modeling Parking Behavior Under Uncertainty: A Static Game Theoretic versus a Sequential Neo-additive Capacity Modeling Approach. Networks and Spatial Economics 13, 327350.

He, F., Yin, Y., Chen, Z., Zhou, J., 2015. Pricing of parking games with atomic players. Transportation Research Part B: Methodological 73, 1-12.

Hobbs, B., Metzler, C., Pang, J.S., 2000. Strategic gaming analysis for electric power systems: an MPEC approach IEEE Transactions on Power Systems 15, 638-645.

Hu, J., Mitchell, J., Pang, J., Bennett, K., Kunapuli, G., 2008. On the Global Solution of Linear Programs with Linear Complementarity Constraints. SIAM Journal on Optimization 19, 445-471.

Hu, J., Mitchell, J.E., Pang, J.S., Yu, B., 2011. On linear programs with linear complementarity constraints. Journal of Global Optimization 53, 29-51.

Izmailov, A., Solodov, M., 2004. Newton-Type Methods for Optimization Problems without Constraint Qualifications. SIAM Journal on Optimization 15, 210-228.

Lam, W.H.K., Tam, M.L., Yang, H., Wong, S.C., 1999. Balance of demand and supply of parking spaces.

Liu, W., Yang, H., Yin, Y., 2014a. Expirable parking reservations for managing morning commute with parking space constraints. Transportation Research Part C: Emerging Technologies 44, 185-201.

Liu, W., Yang, H., Yin, Y., Zhang, F., 2014b. A novel permit scheme for managing parking competition and bottleneck congestion. Transportation Research Part C: Emerging Technologies 44, 265-281.

Lu, Y., 2007. Robust transportation network design under user equilibrium. Thesis. Massachusetts Institute of Technology.

Luo, Z.Q., Pang, J.S., Ralph, D., 1996. Mathematical programs with equilibrium constraints. Cambridge University Press.

Mackowski, D., Bai, Y., Ouyang, Y., 2015. Parking space management via dynamic performance-based pricing. Transportation Research Part C: Emerging Technologies 59, 66-91.

Pang, J.S., Fukushima, M., 2005. Quasi-variational inequalities, generalized Nash equilibria, and multi-leader-follower games. Computational Management Science 2, 21-56.

Panja, B., Schneider, B., Meharia, P., 2011. Wirelessly Sensing Open Parking Spaces : Accounting and Management of Parking Facility, in: AMCIS 2011 Proceedings - All Submissions, p. 270.

Park, W.J., Kim, B.S., Seo, D.E., Kim, D.S., Lee, K.H., 2008. Parking space detection using ultrasonic sensor in parking assistance system, in: 2008 IEEE Intelligent Vehicles Symposium, pp. 1039-1044.

Pierce, G., Shoup, D., 2013. Getting the Prices Right. Journal of the American Planning Association 79, 67-81.

Powell, W., RuszczyÅski, A., Topaloglu, H., 2004. Learning Algorithms for Separable Approximations of Discrete Stochastic Optimization Problems. Mathematics of Operations Research 29, 814-836.

Powell, W.B., 2011. Approximate Dynamic Programming: Solving the Curses of Dimensionality. 2nd ed. ed., John Wiley \& Sons.

Qian, Z.S., Rajagopal, R., 2014a. Optimal dynamic parking pricing for morning commute considering expected cruising time. Transportation Research Part C: Emerging Technologies 48, 468-490.

Qian, Z.S., Rajagopal, R., 2014b. Optimal occupancy-driven parking pricing under demand uncertainties and traveler heterogeneity: A stochastic control approach. Transportation Research Part B: Methodological 67, 144-165.

Qian, Z.S., Rajagopal, R., 2015. Optimal dynamic pricing for morning commute parking. Transportmetrica A: Transport Science 11, 291-316.

Qian, Z.S., Xiao, F.E., Zhang, H.M., 2011. The economics of parking provision for the morning commute. Transportation Research Part A: Policy and Practice 45, 861-879.

Qian, Z.S., Xiao, F.E., Zhang, H.M., 2012. Managing morning commute traffic with parking. Transportation Research Part B: Methodological 46, 894-916.

Rodrigues, H.S., Monteiro, M.T.T., 2006. Solving Mathematical Programs with Complementarity Constraints with Nonlinear Solvers, in: Seeger, P.A. (Ed.), Recent Advances in Optimization. Springer Berlin Heidelberg. number 563 in Lecture Notes in Economics and Mathematical Systems, pp. 415-424.

San Francisco Municipal Transportation Agency, 2014. SFpark: Putting Theory into Practice. http://sfpark . org/ 
resources/docs_pilotsummary. Accessed: 01/10/2016.

Sheffi, Y., 1985. Urban Transportation Networks: Equilibrium Analysis With Mathematical Programming Methods. Prentice Hall, Englewood Cliffs, N.J.

Shoup, D., 2005. The High Cost of Free Parking. Planners Press, American Planning Association.

Sutton, R.S., Barto, A.G., 1998. Reinforcement Learning: An Introduction. MIT Press.

TCRP, 2005. Parking Pricing and Fees, in: Traveler Response to Transportation System Changes Handbook. 3 ed. Transportation Research Board. chapter 13.

Yang, H., Bell, M.G.H., 2001. Transport bilevel programming problems: recent methodological advances. Transportation Research Part B: Methodological 35, 1-4.

Yang, H., Liu, W., Wang, X., Zhang, X., 2013. On the morning commute problem with bottleneck congestion and parking space constraints. Transportation Research Part B: Methodological 58, 106-118.

Zhang, X., Huang, H.J., Zhang, H.M., 2008. Integrated daily commuting patterns and optimal road tolls and parking fees in a linear city. Transportation Research Part B: Methodological 42, 38-56.

Zhang, X., Yang, H., Huang, H.J., 2011. Improving travel efficiency by parking permits distribution and trading. Transportation Research Part B: Methodological 45, 1018-1034. 


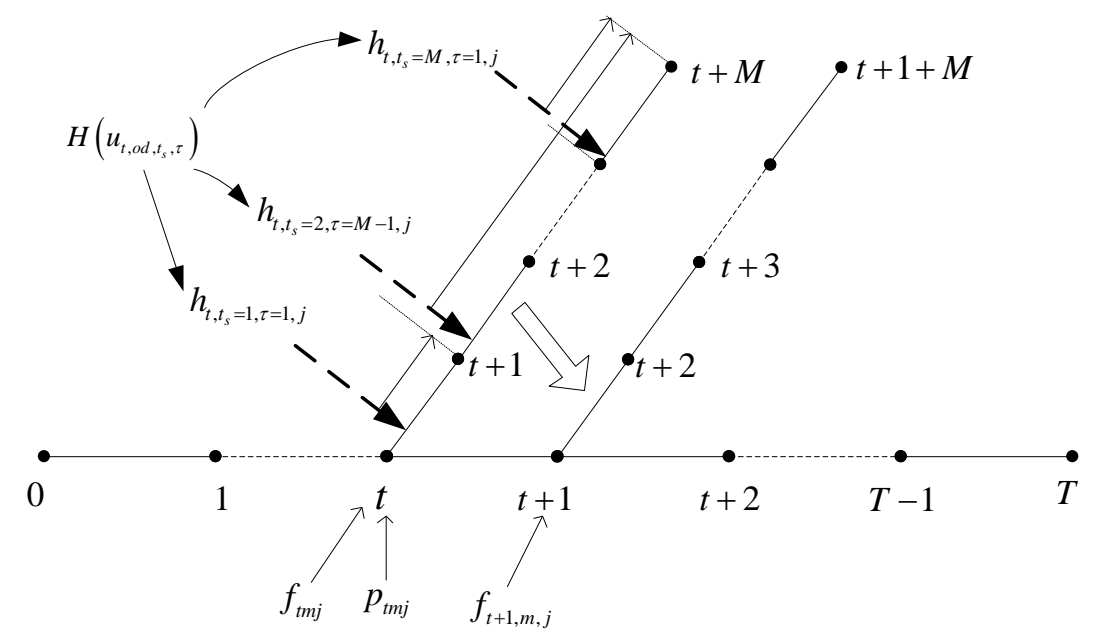

Figure 1: Illustration of the dynamic parking pricing and reservation problem

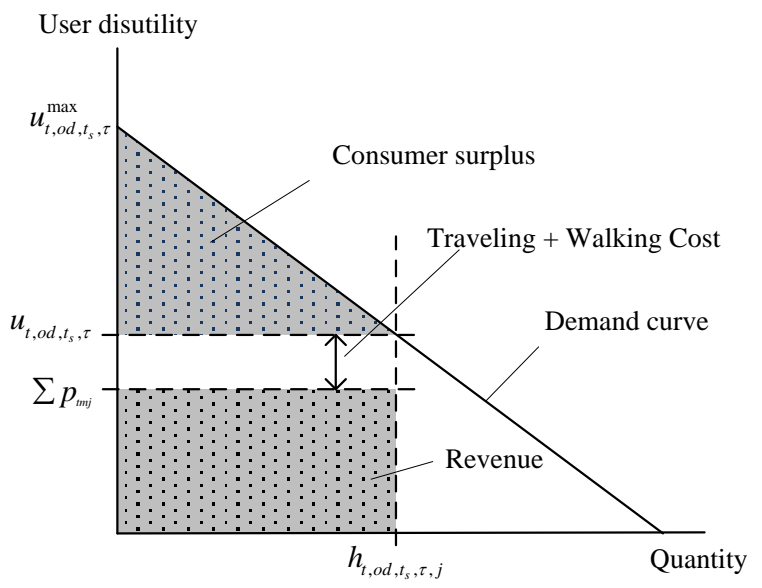

Figure 2: Illustration of the consumer surplus in the parking pricing problem 


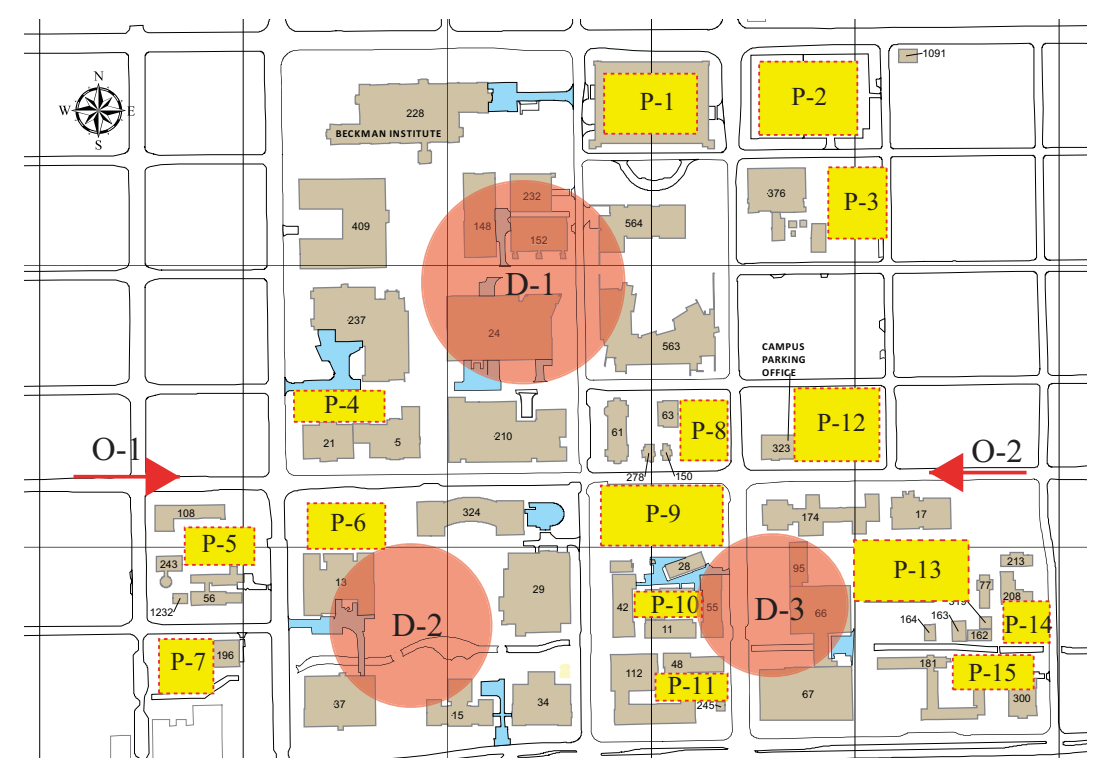

Figure 3: Campus parking map

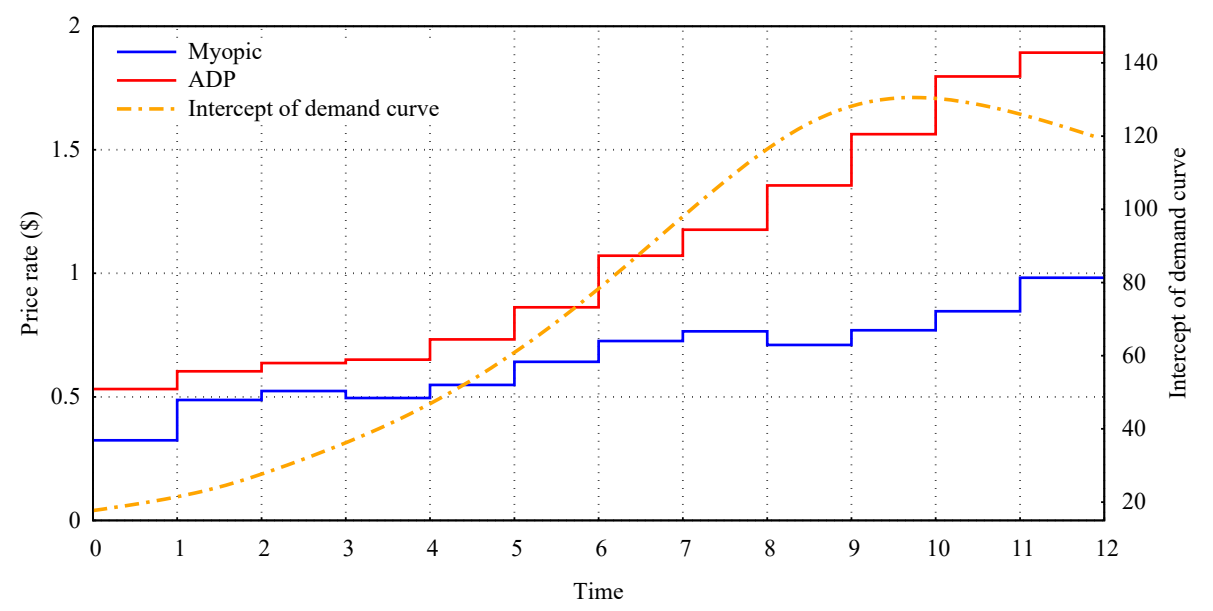

Figure 4: Average price rate for increasing demand pattern $\left(\alpha_{1}=0.5, \alpha_{2}=0.5\right)$ 


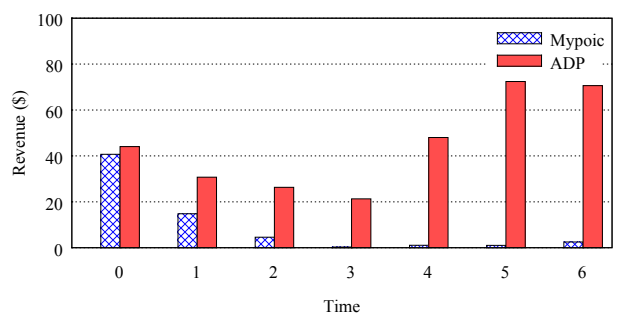

(a) Revenue

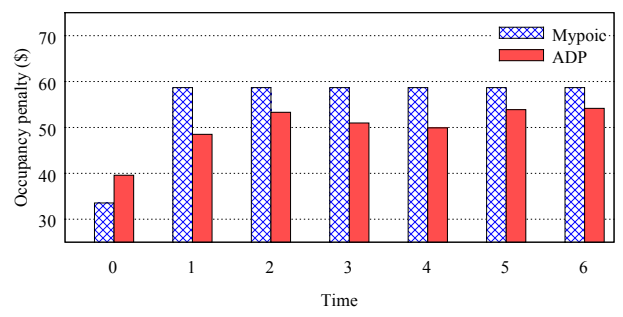

(c) Occupancy imbalance penalty

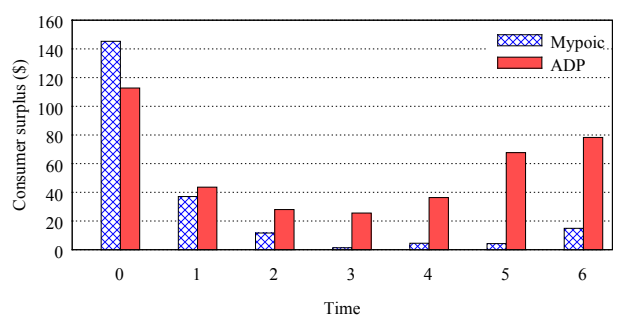

(b) Consumer surplus

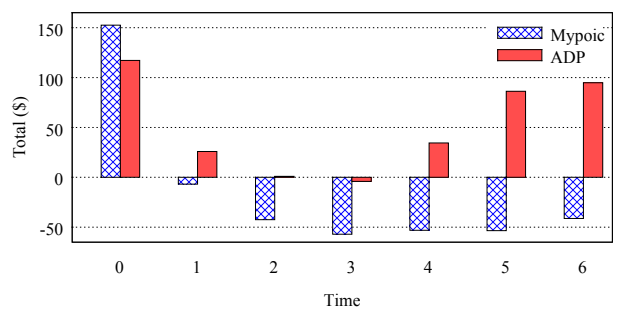

(d) Total objective function value

Figure 5: Comparison of objective function values at each stage $\left(\alpha_{1}=0.5, \alpha_{2}=0.5\right)$

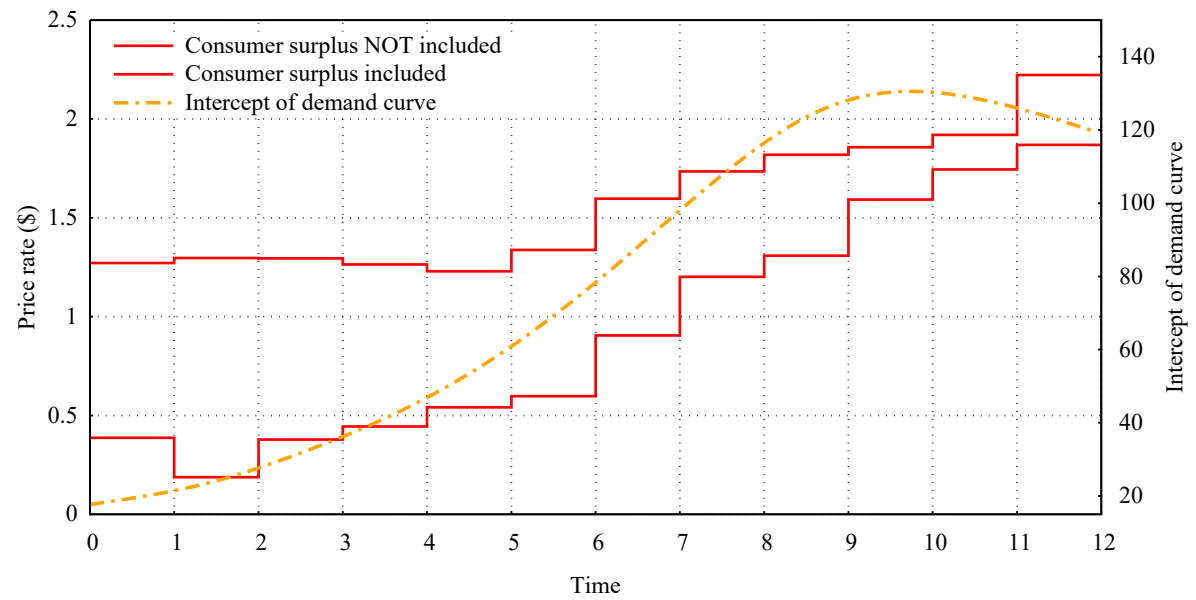

Figure 6: Effect of consumer surplus over price rate $\left(\alpha_{1}=1, \alpha_{2}=0\right)$ 


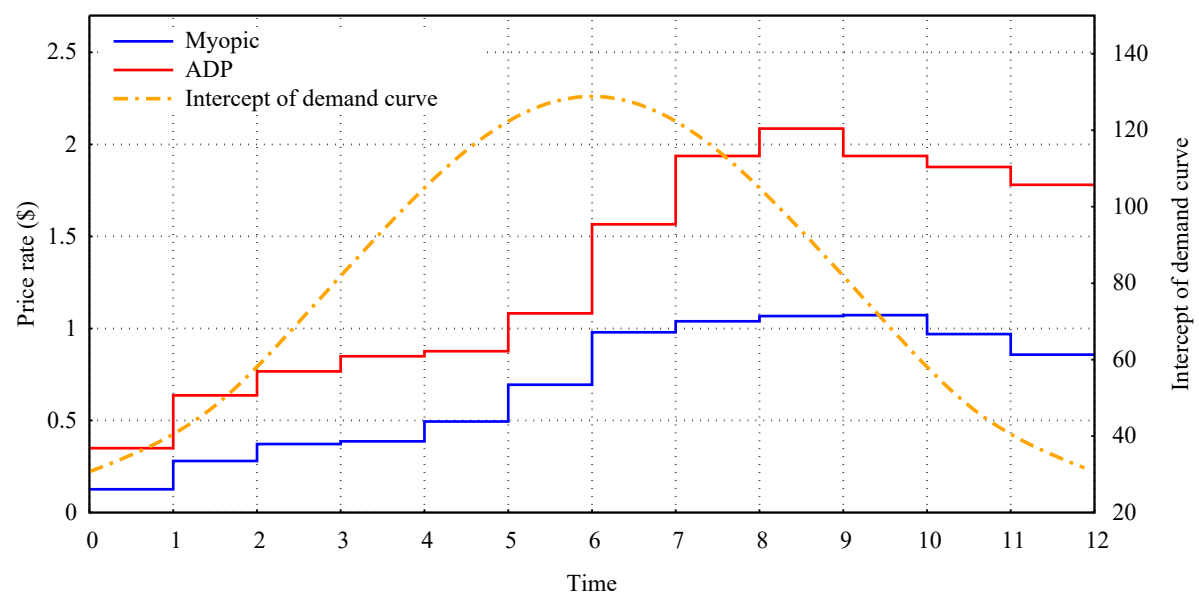

Figure 7: Average price rate for bell shape demand pattern $\left(\alpha_{1}=0.5, \alpha_{2}=0.5\right)$

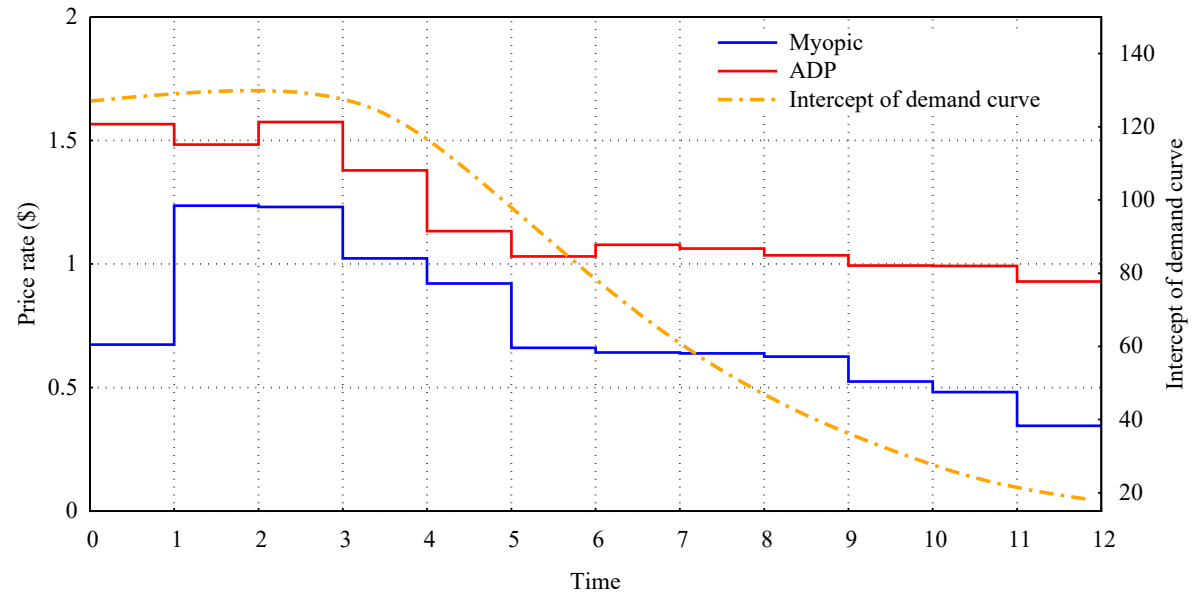

Figure 8: Average price rate for decreasing demand pattern $\left(\alpha_{1}=0.5, \alpha_{2}=0.5\right)$ 


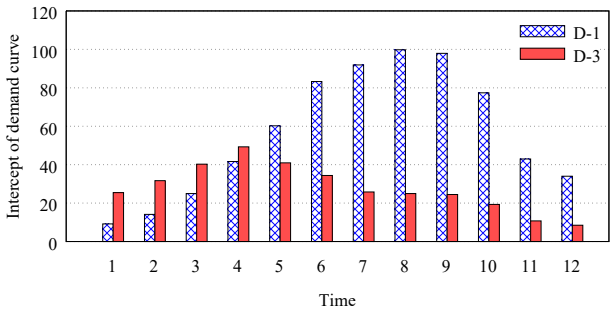

(a) Demand patterns for D-1 and D-3

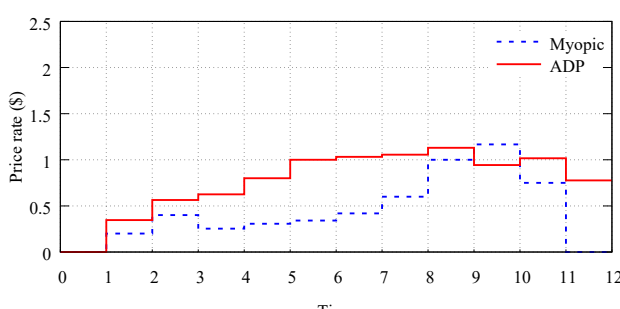

(c) Average price rate for the parking lots close to D-3 but far from D-1

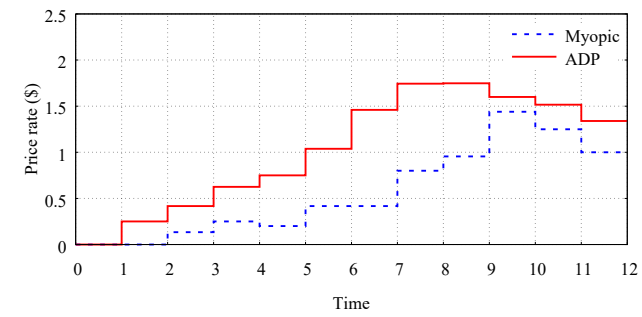

(b) Average price rate for the parking lots close to D-1 but far from D-3

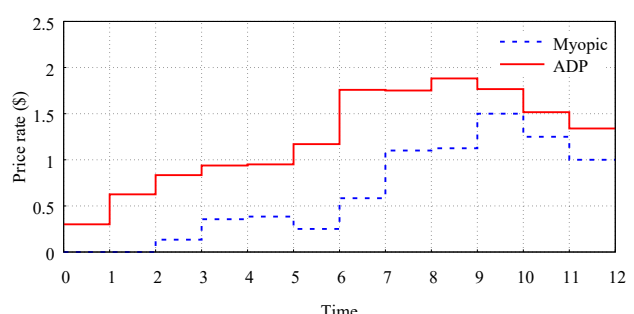

(d) Average price rate for the parking lots close to both D-1 and D-3

Figure 9: Effect of spatial and temporal disparity in parking demand ( $\alpha_{1}=1, \alpha_{2}=0$ ) 


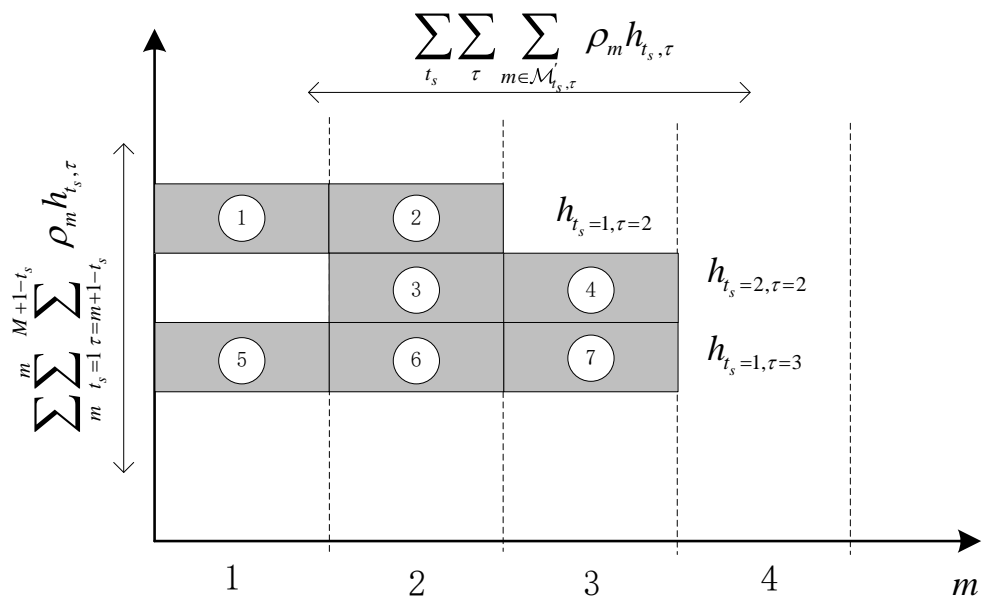

Figure A.10: Graphic illustration of the proposition 
Table 1: Comparison results between ADP and myopic policy

\begin{tabular}{cccccc}
\hline \multirow{2}{*}{$\boldsymbol{\alpha}_{\mathbf{1}}$} & $\boldsymbol{\alpha}_{\mathbf{2}}$ & $\begin{array}{c}\text { CPU time } \\
\text { (sec.) }\end{array}$ & \multicolumn{3}{c}{ Total objective value } \\
\cline { 4 - 6 } & & & Myopic & ADP & Change \\
\hline 1.0 & 0.0 & 5,808 & 586.4 & 1480.0 & $155 \%$ \\
0.9 & 0.1 & 5,908 & 554.2 & 1279.6 & $131 \%$ \\
0.8 & 0.2 & 5,730 & 308.5 & 945.2 & $206 \%$ \\
0.7 & 0.3 & 6,258 & 153.5 & 775.5 & $405 \%$ \\
0.6 & 0.4 & 7,922 & 106.0 & 609.0 & $475 \%$ \\
0.5 & 0.5 & 7,852 & -101.6 & 355.4 & $450 \%$ \\
0.4 & 0.6 & 8,542 & -237.3 & 132.6 & $156 \%$ \\
0.3 & 0.7 & 7,848 & -409.6 & -83.6 & $80 \%$ \\
0.2 & 0.8 & 7,276 & -528.1 & -250.7 & $53 \%$ \\
0.1 & 0.9 & 7,552 & -604.5 & -428.4 & $29 \%$ \\
0.0 & 1.0 & 4,270 & -727.0 & -618.6 & $15 \%$ \\
\hline
\end{tabular}

Table 2: Results of economic surplus and occupancy balance objectives

\begin{tabular}{|c|c|c|c|c|c|c|c|}
\hline \multirow[t]{2}{*}{$\alpha_{1}$} & \multirow[t]{2}{*}{$\alpha_{2}$} & \multicolumn{3}{|c|}{ Economic surplus } & \multicolumn{3}{|c|}{$\begin{array}{c}\text { Occupancy } \\
\text { imbalance penalty }\end{array}$} \\
\hline & & Myopic & ADP & Change & Myopic & ADP & Change \\
\hline 1.0 & 0.0 & 586.4 & 1480.0 & $155 \%$ & 0.0 & 0.0 & - \\
\hline 0.9 & 0.1 & 644.9 & 1359.8 & $111 \%$ & 90.6 & 80.2 & $11 \%$ \\
\hline 0.8 & 0.2 & 470.7 & 1092.1 & $132 \%$ & 162.2 & 146.9 & $9 \%$ \\
\hline 0.7 & 0.3 & 416.9 & 1018.2 & $144 \%$ & 263.3 & 242.6 & $8 \%$ \\
\hline 0.6 & 0.4 & 448.6 & 924.5 & $106 \%$ & 342.6 & 315.5 & $8 \%$ \\
\hline 0.5 & 0.5 & 284.0 & 705.7 & $148 \%$ & 385.6 & 350.3 & $9 \%$ \\
\hline 0.4 & 0.6 & 313.9 & 643.6 & $105 \%$ & 551.2 & 511.0 & $7 \%$ \\
\hline 0.3 & 0.7 & 193.7 & 447.9 & $131 \%$ & 603.3 & 531.5 & $12 \%$ \\
\hline 0.2 & 0.8 & 115.9 & 307.2 & $165 \%$ & 644.0 & 557.9 & $13 \%$ \\
\hline 0.1 & 0.9 & 57.5 & 159.3 & $177 \%$ & 662.0 & 587.7 & $11 \%$ \\
\hline 0.0 & 1.0 & 0.0 & 0.0 & - & 727.0 & 618.6 & $15 \%$ \\
\hline
\end{tabular}

Table 3: Test results for the use of consumer surplus $\left(\alpha_{1}=1, \alpha_{2}=0\right)$

\begin{tabular}{lccc}
\hline & Revenue & Consumer surplus & Total \\
\hline Consumer surplus NOT included & 925.3 & 362.6 & 1287.9 \\
Consumer surplus included & 697.6 & 800.4 & 1480.0 \\
\hline
\end{tabular}


Table 4: Results for different demand patterns $\left(\alpha_{1}=0.5, \alpha_{2}=0.5\right)$

\begin{tabular}{|c|c|c|c|c|c|c|}
\hline \multirow{2}{*}{$\begin{array}{l}\text { Demand } \\
\text { pattern }\end{array}$} & \multicolumn{2}{|c|}{ Economic surplus } & \multicolumn{2}{|c|}{$\begin{array}{c}\text { Occupancy } \\
\text { imbalance penalty }\end{array}$} & \multicolumn{2}{|c|}{ Total } \\
\hline & Myopic & ADP & Myopic & ADP & Myopic & ADP \\
\hline Increasing & 284.0 & 705.7 & 385.6 & 350.3 & -101.6 & 355.4 \\
\hline Bell shape & 174.7 & 679.5 & 364.9 & 346.5 & -190.2 & 333.0 \\
\hline Decreasing & 861.5 & 924.7 & 280.5 & 274.3 & 581.0 & 650.4 \\
\hline
\end{tabular}

Table 5: Results for spatial and temporal disparity in demand $\left(\alpha_{1}=0.5, \alpha_{2}=0.5\right)$

\begin{tabular}{lcccc}
\hline & Revenue & Consumer surplus & $\begin{array}{c}\text { Occupancy } \\
\text { imbalance penalty }\end{array}$ & Total \\
\hline Myopic & 58.2 & 253.0 & 368.9 & -57.6 \\
ADP & 261.1 & 535.4 & 298.9 & 497.6 \\
\hline
\end{tabular}




\begin{abstract}
Despite rapid advances of information technologies for intelligent parking systems, it remains a challenge to optimally manage limited parking resources in busy urban neighborhoods. In this paper, we use dynamic location-dependent parking pricing and reservation to improve system-wide performance of an intelligent parking system. With this system, the parking agency is able to decide the spatial and temporal distribution of parking prices to achieve a variety of objectives, while drivers with different origins and destinations compete for limited parking spaces via online reservation. We develop a multi-period non-cooperative bi-level model to capture the complex interactions among the parking agency and multiple drivers, as well as a non-myopic approximate dynamic programming (ADP) approach to solve the model. It is shown with numerical examples that the ADP-based pricing policy consistently outperforms alternative policies in achieving greater performance of the parking system, and shows reliability in handling the spatial and temporal variations in parking demand.
\end{abstract}




\section{Keywords:}

Parking management, Dynamic pricing, Approximate dynamic programming, Equilibrium, MPEC 


\section{List of Figures}

1 Illustration of the dynamic parking pricing and reservation problem $\ldots \ldots 23$

2 Illustration of the consumer surplus in the parking pricing problem . . . . . 23

3 Campus parking map ... . . . . . . . . . . . . . 24

4 Average price rate for increasing demand pattern $\left(\alpha_{1}=0.5, \alpha_{2}=0.5\right) \quad \ldots \ldots 24$

5 Comparison of objective function values at each stage $\left(\alpha_{1}=0.5, \alpha_{2}=0.5\right) \quad \ldots 25$

6 Effect of consumer surplus over price rate $\left(\alpha_{1}=1, \alpha_{2}=0\right) \ldots \ldots 25$

7 Average price rate for bell shape demand pattern $\left(\alpha_{1}=0.5, \alpha_{2}=0.5\right) \ldots \ldots 26$

8 Average price rate for decreasing demand pattern $\left(\alpha_{1}=0.5, \alpha_{2}=0.5\right) \ldots \ldots 26$

9 Effect of spatial and temporal disparity in parking demand $\left(\alpha_{1}=1, \alpha_{2}=0\right) \quad \ldots 27$

A.10 Graphic illustration of the proposition . . . . . . . . . . . . 28 


\section{List of Tables}

Comparison results between ADP and myopic policy . . . . . . . . . . . 29

Results of economic surplus and occupancy balance objectives . . . . . . . . 29

Test results for the use of consumer surplus $\left(\alpha_{1}=1, \alpha_{2}=0\right) \quad \ldots \ldots \ldots$

Results for different demand patterns $\left(\alpha_{1}=0.5, \alpha_{2}=0.5\right) \ldots \ldots \ldots \ldots$

5 Results for spatial and temporal disparity in demand $\left(\alpha_{1}=0.5, \alpha_{2}=0.5\right) \ldots \ldots 30$ 